\title{
Functionalized boron-dipyrromethenes and their applications
}

\author{
This article was published in the following Dove Press journal: \\ Reports in Organic Chemistry \\ 18 January 2016 \\ Number of times this article has been viewed
}

\author{
Vellanki Lakshmi \\ Ritambhara Sharma \\ Mangalampalli Ravikanth \\ Department of Chemistry, Indian \\ Institute of Technology Bombay, \\ Mumbai, India
}

\begin{abstract}
Boron-dipyrromethenes/ $\mathrm{BF}_{2}$-dipyrrins (BODIPYs) are highly fluorescent dyes with a wide range of applications in various fields because of their attractive photophysical properties. One of the salient features of BODIPYs is that the properties of the BODIPY can be fine-tuned at will by selectively introducing the substituent(s) at the desired location(s) of the BODIPY. The BODIPYs have several potential sites where the functional groups can be introduced and the functionalized BODIPYs can be used as building blocks to synthesize the desired BODIPY derivatives with interesting features. In this review, we presented the synthesis of different types of functionalized BODIPYs where the functional group(s) were introduced directly at the meso-carbon, at the pyrrole carbons of the BODIPY core as well as at the boron center and discussed their applications toward synthesis of simple substituted BODIPYs to complex BODIPY based systems.
\end{abstract}

Keywords: boron-dipyrromethenes, functionalization, electronic properties, sterically crowded, fluorescence, application

\section{Introduction}

Fluorescent dyes are very useful for visualizing biomolecules in vitro and in vivo. ${ }^{1-3}$ The most important criteria for fluorescent dyes is their ability to overcome biological barriers and accumulate and localize at the subcellular targets. Accordingly, significant efforts have been made to develop fluorescent probes for bioimaging applications. ${ }^{4-7}$ The most widely used fluorescent probes are coumarin, fluorescein, boron-dipyrromethenes/ $\mathrm{BF}_{2}$-dipyrrins (BODIPYs), rhodamine, and cyanine dyes. ${ }^{8-10}$ Among these fluorescent probes, BODIPYs ${ }^{11}$ have played a pivotal role in fluorescent probe development and it is one of the most exploited fluorophores because of the following features: 1) sharp absorption and fluorescence in the visible and near-infrared region depending on substituents; 2) large absorption coefficients, high fluorescence quantum yields, and reasonably long singlet state lifetimes; 3) easy to synthesize; 4) chemical, thermal, and photostability; and 5) relative insensitivity to environmental perturbations such as solvent and $\mathrm{pH}$. Besides their extensive applications as fluorescent probes in biology, the BODIPY dyes are also widely used as cation and anion sensors, drug delivery agents, fluorescent switches, electroluminescent films, laser dyes, light harvesters, and sensitizers for solar cells. ${ }^{12-16}$ The wide applications of BODIPY dyes are because of their excellent physico-chemical properties combined with their ease of synthesis. Furthermore, the photophysical properties of BODIPYs can be fine-tuned by changing the substituents of different electron densities or by manipulating the conjugation length or by chemical modification at various positions of BODIPY dyes. ${ }^{17}$ Such modifications can be carried out conveniently on BODIPY dyes because these dyes are amenable
Correspondence: Mangalampalli Ravikanth Department of Chemistry, Indian Institute of Technology Bombay, Powai, Mumbai 400 076, India

Email ravikanth@chem.iitb.ac.in
Reports in Organic Chemistry 2016:6 I-24 (c) (i) (2) 2016 Lakshmi et al. This work is published by Dove Medical Press Limited, and licensed under Creative Commons Attribution - Non Commercial (unported, v3.0) permission from Dove Medical Press Limited, provided the work is properly attributed. Permissions beyond the scope of the License are administered by Dove Medical Press Limited. Information on how to request permission may be found at: http://www.dovepress.com/permissions.php 
for functionalization at all positions and the functionalized BODIPYs are suitable synthons to prepare a wide variety of BODIPY derivatives with desired photophysical properties. Thus, the functional group(s) can be introduced at the various positions of BODIPYs such as at the meso-aryl group; directly at the meso group; at the pyrrole carbon(s) BODIPY core, and also at the B(III) center. In recent times, several reviews have appeared on various aspects of BODIPY dyes ${ }^{18-22}$ including the reviews on BODIPY based probes for the fluorescence imaging of biomolecules in living cells; fluorescent indicators for $\mathrm{pH}$, metal ions, anions, biomolecules, reactive oxygen and nitrogen species, redox potential and chemical reactions and photosensitizers for photodynamic therapy along with reviews on synthesis and properties of BODIPY dyes. Interestingly, a perusal of literature reveals that there is no dedicated review on functionalized BODIPYs and their applications to prepare novel BODIPY based complex systems having potential applications in various research fields. In this review, we focus on various functionalized BODIPYs and their applications in synthesis of selective BODIPY based complex systems. This review is limited to the synthesis of functionalized BODIPYs and how the functionalized BODIPYs were used for the synthesis of some complex BODIPY based systems but their applications in materials, biology, and medicinal fields were not discussed here. Readers interested in BODIPY applications can refer to the excellent reviews that have recently appeared in the literature. ${ }^{12-16}$ Since the functional groups were introduced either on dipyrromethane I or directly on BODIPY II and the numbering of the positions for these two are different, we presented the numbering on dipyrromethane I and BODIPY II in Figure 1. The functionalized BODIPYs are classified based on the position at the BODIPY systems as discussed below.

\section{Meso-functionalized BODIPYs Meso-aryl functionalized BODIPYs}

BODIPYs containing a functionalized aryl group at the meso-position is the most desirable fluorophore for the
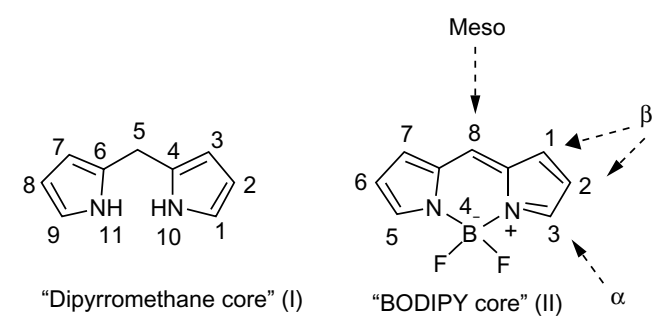

Figure I Dipyrromethane and boron-dipyrromethene skeletons and their International Union of Pure and Applied Chemistry numbering figure.

Abbreviation: BODIPY, boron-dipyrromethene $/ \mathrm{BF}_{2}$-dipyrrin. construction of complex BODIPY fluorophores ${ }^{18-22}$ because of the straightforward synthesis from functionalized aromatic aldehydes. Meso-aryl functionalized BODIPYs are highly desirable building blocks to synthesize simple BODIPY based systems ${ }^{18-22}$ to complex light harvesting arrays, ${ }^{23}$ photonic wires, ${ }^{24}$ and opto-electronic gates. ${ }^{25,26}$ The meso-aryl functionalized BODIPYs can be prepared easily by following the well-established Lindsey's ${ }^{27}$ traditional Lewis acid catalyzed approach. In Lindsey's approach, the synthesis of meso-aryl functionalized BODIPYs involves the condensation reaction between $\alpha$-free pyrrole and functionalized aryl aldehydes in the presence of a catalytic amount of Lewis acid to obtain dipyrromethane which is on subsequent oxidation by DDQ (2,3-dichloro-5,6-dicyano-1,4-benzoquinone) to dipyrromethene followed by $\mathrm{BF}_{2}$ complexation using $\mathrm{BF}_{3} \cdot \mathrm{OEt}_{2}$ under basic conditions to yield meso-aryl BODIPYs (Figure 2). The meso-aryl functionalized BODIPYs are very stable to carry out functional group inter-conversions to afford other meso-aryl functionalized BODIPYs where the functionalized aryl aldehydes are not commercially available. Thus, this synthetic protocol gives access to prepare several meso-aryl functionalized BODIPYs that have been used for the construction of several complex BODIPY based systems. There are several reviews available on BODIPY based systems using meso-aryl functionalized BODIPYs. ${ }^{28,29}$ However, here we mainly focus on functionalized BODIPYs where the functional group was directly present at the meso carbon of BODIPY which was subsequently used for the synthesis of other functionalized BODIPYs and complex substituted BODIPYs.

\section{Meso-thiomethyl BODIPY}

Although Lindsey's methodology ${ }^{27}$ gives access to synthesize several complex BODIPY based systems, there are certain drawbacks with this approach that include laborious purifications, the separate synthesis of the starting aldehydes if aldehydes are not commercially available, and incompatibility of the functional groups on the aryl ring of the aryl aldehyde toward exposure to strong Lewis acids and DDQ oxidation. These limitations of Lindsey's protocol challenged researchers to look for alternate routes to prepare the required meso-substituted BODIPYs. In 2006, Goud et $\mathrm{al}^{30}$ reported the synthesis 8-thiomethyl BODIPY 3 which acted as a key synthon to prepare different mesosubstituted BODIPYs via Liebeskind-Srogl cross-coupling reaction $^{31}$ and an $\mathrm{S}_{\mathrm{N}}$ Ar-like process.

The Biellmann's BODIPY was prepared in a few steps. In the first step, the pyrrole was reacted with thiophosgene (Figure 3 ) to produce thioketone. ${ }^{30}$ In the subsequent steps, 


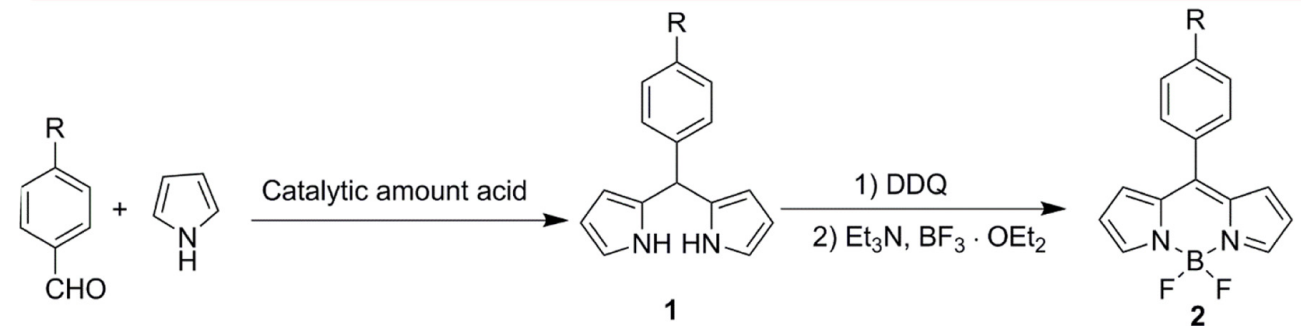

Figure 2 Synthesis of meso-aryl boron-dipyrromethene complexes 2 .

Note: $\mathrm{R}=\mathrm{NO}_{2}, \mathrm{NH}_{2}, \mathrm{OH}, \mathrm{Cl}, \mathrm{Br}, \mathrm{I}, \mathrm{F}, \mathrm{CN}$ etc.

Abbreviation: DDQ, 2,3-dichloro-5,6-dicyanobenzoquinone.

the thioketone was reacted with $\mathrm{CH}_{3} \mathrm{I}$ followed by treatment with tertiary amine and complexation with $\mathrm{BF}_{3} \cdot \mathrm{OEt}_{2}$ to produce 8-thiomethyl BODIPY 3. Peña-Cabrera et $\mathrm{al}^{32-37}$ used 8-thiomethyl BODIPY 3 to prepare a series of mesosubstituted BODIPYs under Liebeskind-Srogl cross-coupling reaction conditions. ${ }^{31}$ The 8-thiomethyl BODIPY 3 was treated with aryl, heteroaryl, alkenyl and organometallic boronic acids (Figure 4) in the presence of a catalytic amount of $\operatorname{Pd}(0)$ /trifurylphosphine and a stoichiometric amount of $\mathrm{Cu}(\mathrm{I})$-2-thienylcarboxylate under neutral conditions and prepared corresponding meso-substituted BODIPYs 4-9. $32-37$ The reaction also works efficiently if one uses organostannanes instead of boronic acids under the same $\mathrm{Pd}(0)$ coupling conditions. ${ }^{38}$ They have used this methodology to prepare different types of meso-aryl functionalized BODIPYs like 10 and the functionalized meso-aryl group was used as handle for additional elaboration. For example, BODIPY containing meso-bromoaryl group 8 was used to couple with different substituted boronic acids under Suzuki coupling conditions to prepare meso-polyaryl 15 or meso-polyarylamine BODIPY hybrids 15 (Figure 4) in decent yields. ${ }^{32}$ BODIPY containing azido functionalized meso-aryl group 9 was used to react with propargyl $\alpha$-D-mannopyranoside under $\mathrm{Cu}$ (I) catalyzed click reaction conditions to afford carbohydrate-BODIPY hybrid $16 .{ }^{33}$ The BODIPY containing meso-alkenyl group 6 was subjected to Michael-type nucleophilic addition by treating it with dodecane-1-thiol in THF (tetrahydrofuran) under mild reaction conditions (Figure 4) to obtain the desired adduct $12 .{ }^{34}$ The meso-alkyl containing BODIPY 13 was also prepared in high yield by $\mathrm{Pd}$-catalyzed reduction of corresponding meso-alkenyl BODIPY $6 .{ }^{34}$

The meso-vinylphenyl substituted BODIPY 7 was subjected to Grubbs catalyzed conditions (Figure 4) to prepare novel BODIPY dyad $14 .^{32}$ Thus, the meso-aryl functionalized/ alkenyl containing BODIPYs open up the synthesis of a wide variety of complex BODIPY systems under mild reaction conditions. Recently the same research group developed a straightforward synthetic route ${ }^{39}$ to directly incorporate 1,3-dicarbonyl nucleophiles such as acetylacetonate into the meso-position of BODIPY 11 by treating 8-(thiomethyl) BODIPY 3 (Figure 5) with 1,3-dicarbonyl derivatives in the presence of $\mathrm{Cu}(\mathrm{I})$ thiophenecarboxylate in stoichiometric amounts at room temperature.

The thiomethyl $\left(-\mathrm{SCH}_{3}\right)$ group at the meso-position showed not only excellent reactivity as a cross-coupled partner but also acted as simple leaving group to prepare $\mathrm{N}-$ and $\mathrm{O}-$ based nucleophiles substituted at the meso-position of BODIPY. Thus, meso-(thiomethyl) group of BODIPY 3 can be displaced in an $\mathrm{S}_{\mathrm{N}} \mathrm{Ar}$-like process by amines to produce mesoamine substituted BODIPYs 17-24 (Figure 5). Goud et al who first prepared meso-(thiomethyl) BODIPY 3 showed $^{30}$ that $-\mathrm{SCH}_{3}$ group can be displaced by reacting 3 with aniline in $\mathrm{CH}_{2} \mathrm{Cl}_{2}$ to form meso-anilino BODIPY 19. Later, OsorioMartínez et al relooked at the reaction and prepared several meso-substituted amino BODIPYs $17-24$ by treating 3 with various substituted amines. ${ }^{40-44}$ The meso-amino BODIPY 23
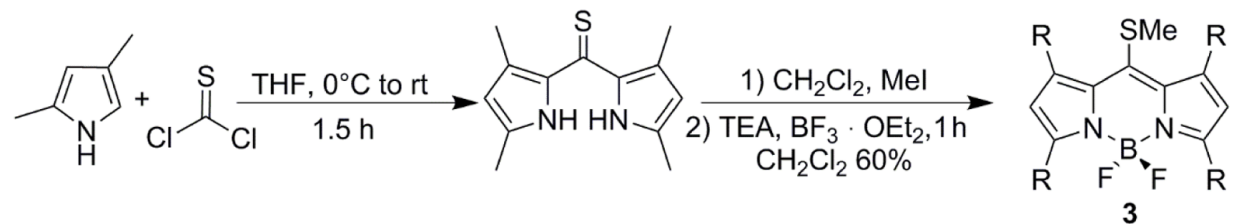

Figure 3 Synthesis of meso-(thiomethyl) BODIPY 3.

Note: $\mathrm{R}=\mathrm{H} / \mathrm{CH}_{3}: 3$

Abbreviations: BODIPY, boron-dipyrromethene/BF 2 -dipyrrin; $h$, hours; $T H F$, tetrahydrofuran; $r t$, room temperature. 


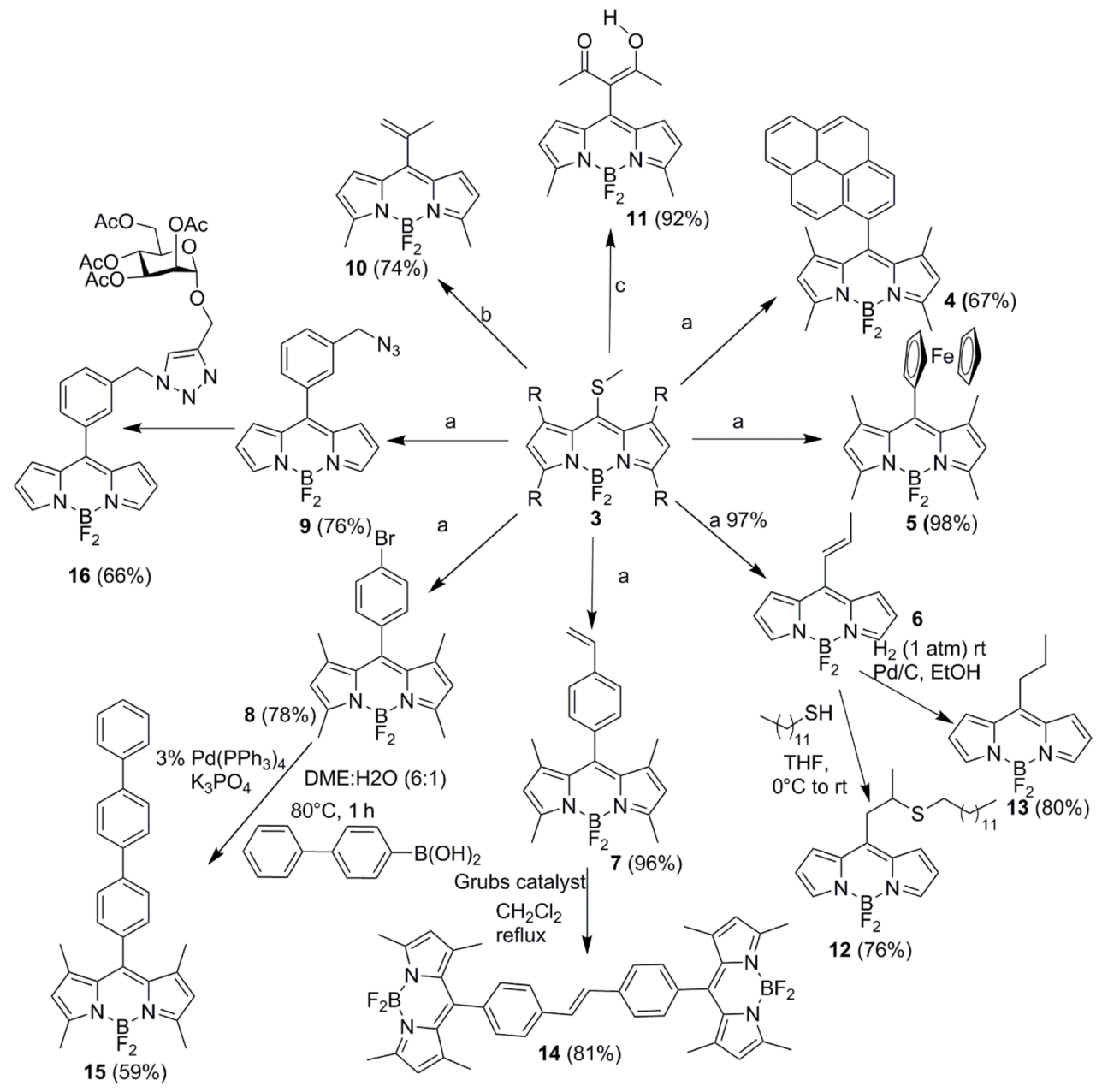

Reaction condition: (a) $2.5 \% \mathrm{Pd}_{2}(\mathrm{dba})_{3}, 7.5 \%$ TFP, 3 equiv CuTc, THF, $55^{\circ} \mathrm{C}, \mathrm{R}-\mathrm{B}(\mathrm{OH})_{2}$

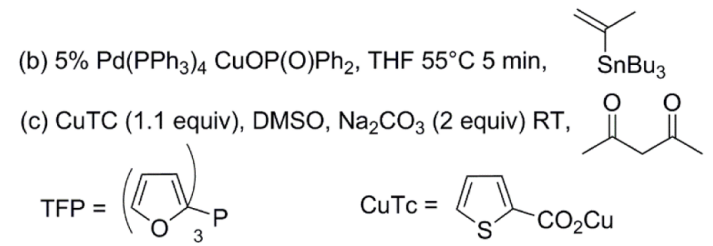

Figure 4 Synthesis of various types of meso-substituted BODIPYs 4-I6 derived from meso-(thiomethyl) BODIPY 3 under Pd catalyzed conditions.

Abbreviations: BODIPY, boron-dipyrromethene/BF 2 -dipyrrin; equiv, equivalent(s); min, minutes; DMSO, dimethyl sulfoxide; atm, atmospheric pressure; THF, tetrahydrofuran; $\mathrm{rt}$, room temperature.

was prepared by treating meso-(thiomethyl) BODIPY 3 with ammonia. The other meso-amino substituted BODIPYs 17-24 (Figure 5) were prepared by treating meso-(thiomethyl) BODIPY 3 with corresponding amines. The meso-substituted amino-BODIPYs 17-24 were also used further to prepare several interesting meso-substituted BODIPY compounds. For example, meso-(propargylamino) BODIPY 18 on reaction with azido-carbohydrates under click reaction conditions (Figure 5) produced BODIPY-carbohydrate hybrid $26 .{ }^{33}$ The meso-methylamino BODIPY 20 on treatment with $\mathrm{CH}_{3} \mathrm{I}$ in the presence of $\mathrm{NaH}$ in THF at $0^{\circ} \mathrm{C}$ produced meso(N,N'-dimethylamino) BODIPY 27. ${ }^{43}$ Ma et al synthesized ${ }^{41}$ a fluorescence resonance energy transfer (FRET) donor connected BODIPY conjugate by treating meso-substituted amino BODIPY 24 with FRET such as fluorescein isothiocyanate and prepared fluorescein isothiocyanate-BODIPY FRET couple 25 (Figure 5) and used for sensing cysteine. Thus, meso-substituted amino BODIPYs gave wide access to prepare several novel meso-substituted amino BODIPYs (Figure 5). The presence of substituted amino group at the meso-position of BODIPYs alters the electronic properties significantly and these meso-amino substituted BODIPYs absorb and emit efficiently in the blue spectral region unlike other meso-aryl BODIPYs which are normally absorbed in 


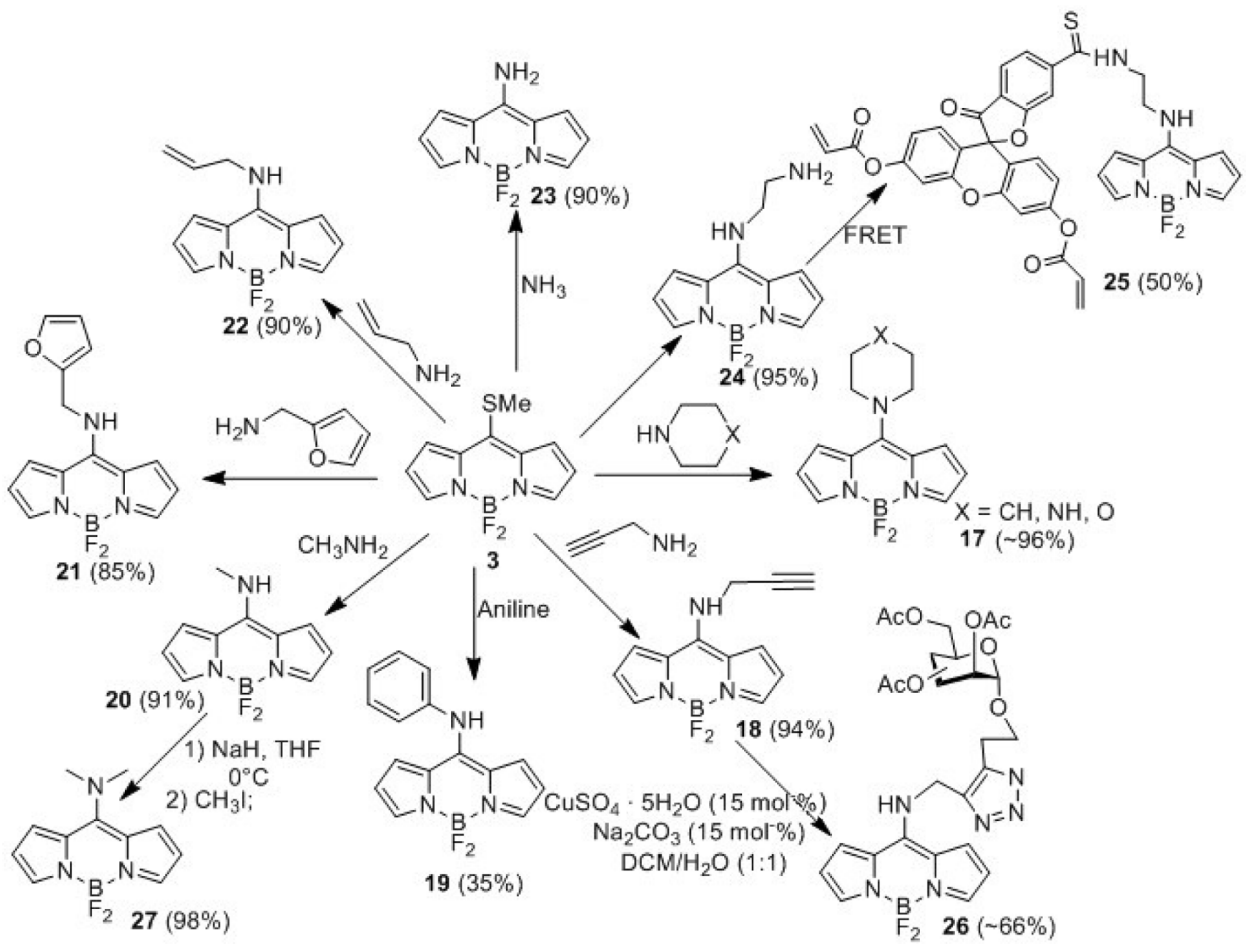

Figure 5 Synthesis of various types of meso-substituted BODIPYs 17-27 derived from meso-(thiomethyl) BODIPY 3 by nucleophilic substitution. Abbreviations: BODIPY, boron-dipyrromethene/ $\mathrm{BF}_{2}$-dipyrrin; $\mathrm{FRET}$, fluorescence resonance energy transfer; THF, tetrahydrofuran.

the blue-green region as well as in red and infra-red region upon slight modifications. Thus, the meso-substituted amino BODIPYs are blue emitting dyes and some of these dyes exhibit very high fluorescence quantum yields, close to unity, and large Stokes shifts, leading to highly efficient tunable laser dyes in the blue part of the spectrum.

The meso-(thiomethyl) BODIPY 3 was also used effectively to prepare meso-alkoxy 31 and meso-aryloxy BODIPYs 29 by treating it with corresponding alcohols (Figure 6) in the presence of a base and $\mathrm{Cu}(\mathrm{I})$ additive. ${ }^{45,46} \mathrm{In}$ addition to simple alcohols, they also succeeded in connecting natural products such as eugenol, menthol, cholesterol 28, and estrone 32 under simple reaction conditions (Figure 6) ${ }^{45}$ These hybrids are highly fluorescent in the blue-green part of the visible region and the BODIPY bearing cholesterol at the meso-position 28 can be used as fluorescence biological marker. Esnal et al also introduced phosphorus ${ }^{46}$ atom at the meso-position of the BODIPY core by treating meso-(thiomethyl) BODIPY 3 with excess of $\mathrm{PPh}_{2} \mathrm{H}$ in THF (Figure 6) and yielded 30 in $61 \%$ yield. Thus, the meso-(thiomethyl) BODIPY 3 allows the introduction of different kinds of $-\mathrm{C},-\mathrm{N}$, and $-\mathrm{O}$ based nucleophiles at the meso-position via Liebeskind-Srogl coupling reaction and $\mathrm{S}_{\mathrm{N}}$ Ar-process. These dyes can absorb and emit from the blue region to visible-near-infrared region depending on the meso-substituent.

\section{Meso-halogenated BODIPYs}

The meso-substituted BODIPYs can also be prepared by using meso-halo BODIPYs. Leen et al reported ${ }^{47}$ the synthesis of meso-halogenated BODIPYs and their applications in the synthesis of meso-substituted BODIPYs via $\mathrm{S}_{\mathrm{N}} \mathrm{Ar}$ process and palladium-catalyzed Suzuki, Stille and Sonogashira crosscoupling reactions. The meso-chloro BODIPY 35 was prepared by reacting the dipyrrolylketone with $\mathrm{POCl}_{3}$ followed by in situ deprotonation by base and complexation with $\mathrm{BF}_{3} \cdot \mathrm{OEt}_{2}$. Similarly, the dipyrrolylketone was reacted (Figure 7) with $\mathrm{POBr}_{3}$ followed by deprotonation and complexation with $\mathrm{BF}_{3} \cdot \mathrm{OEt}_{2}$ in situ to afford meso-bromo BODIPY 33. However, under the same reaction conditions, $\mathrm{PI}_{3}$ was unsuccessful in producing meso-iodo BODIPY 34. They were also unsuccessful to prepare meso-fluoro BODIPY. Leen et al adopted a halogen exchange approach to prepare meso-iodo BODIPY 34. In modified Finkelstein procedure, the meso-chloro BODIPY 35 was stirred in acetone in the presence of sodium iodide (Figure 7) and afforded meso-iodo BODIPY 34 in good yield.

The meso-halo BODIPYs like meso-(thiomethyl) BODIPY 3 are very useful compounds because they are very promising functionalized BODIPYs for the preparation of more complex meso-substituted BODIPY 36-44 analogs via elaboration of the reactive halogen by $\mathrm{S}_{\mathrm{N}} \mathrm{Ar}$ or transition metal catalyzed transformations such as Suzuki, Stille, Heck, 


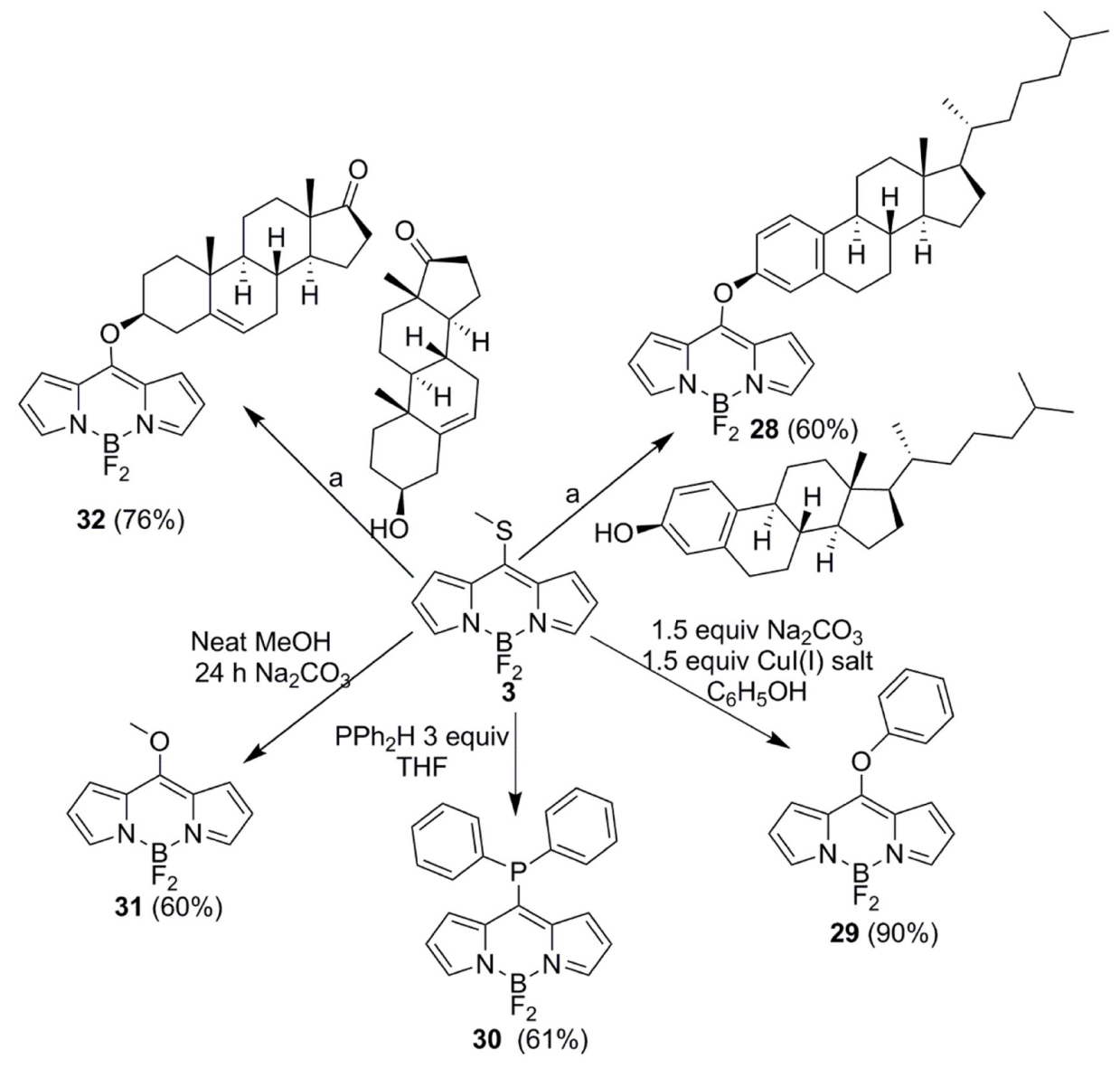

Figure 6 Synthesis of nucleophilic substituted BODIPYs 28-32 derived from meso-thiomethyl BODIPY 3.

Note: (a) 1.5 equiv CuTC, 1.5 equiv. $\mathrm{Na}_{2} \mathrm{CO}_{3}, \mathrm{CH}_{3} \mathrm{CN}, 55^{\circ} \mathrm{C}$.

Abbreviations: $\mathrm{BODIPY}$, boron-dipyrromethene/ $\mathrm{BF}_{2}$-dipyrrin; equiv, equivalent(s); h, hours; THF, tetrahydrofuran.

Negishi, Sonogashira etc as shown in Figure 8. The chloride substituent at the meso-position of BODIPY is an efficient leaving group in $\mathrm{S}_{\mathrm{N}}$ Ar reactions. Meso-chloro BODIPY 35 was also useful to prepare a wide range of heteroatom $(-\mathrm{N}$, $-\mathrm{O}$, and $-\mathrm{S}$ ) meso-substituted derivatives by nucleophilic displacement of the meso-halogen substituent of BODIPY. ${ }^{47-49}$ Several meso-substituted derivatives 36-39 were prepared by stirring meso-halo BODIPY with appropriate nucleophile (N, O, and S-nucleophiles) in the presence of base. The meso-halo BODIPYs were also used to prepare several

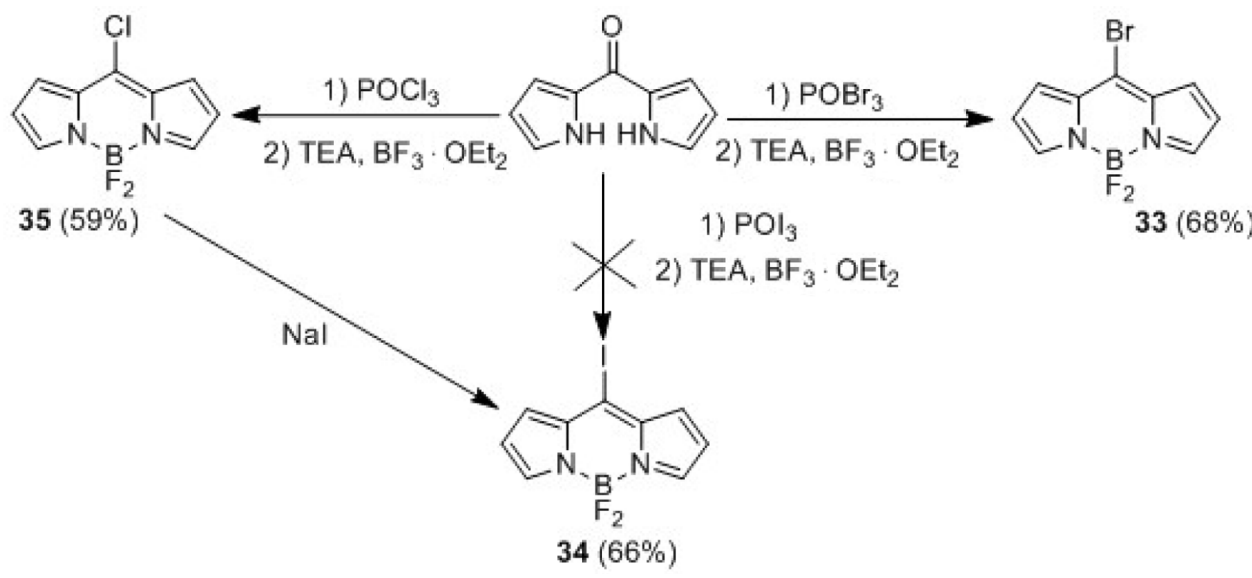

Figure 7 Synthesis of meso-functionalized BODIPYs 33-35.

Abbreviation: BODIPYs, boron-dipyrromethenes/ $\mathrm{BF}_{2}$-dipyrrins. 


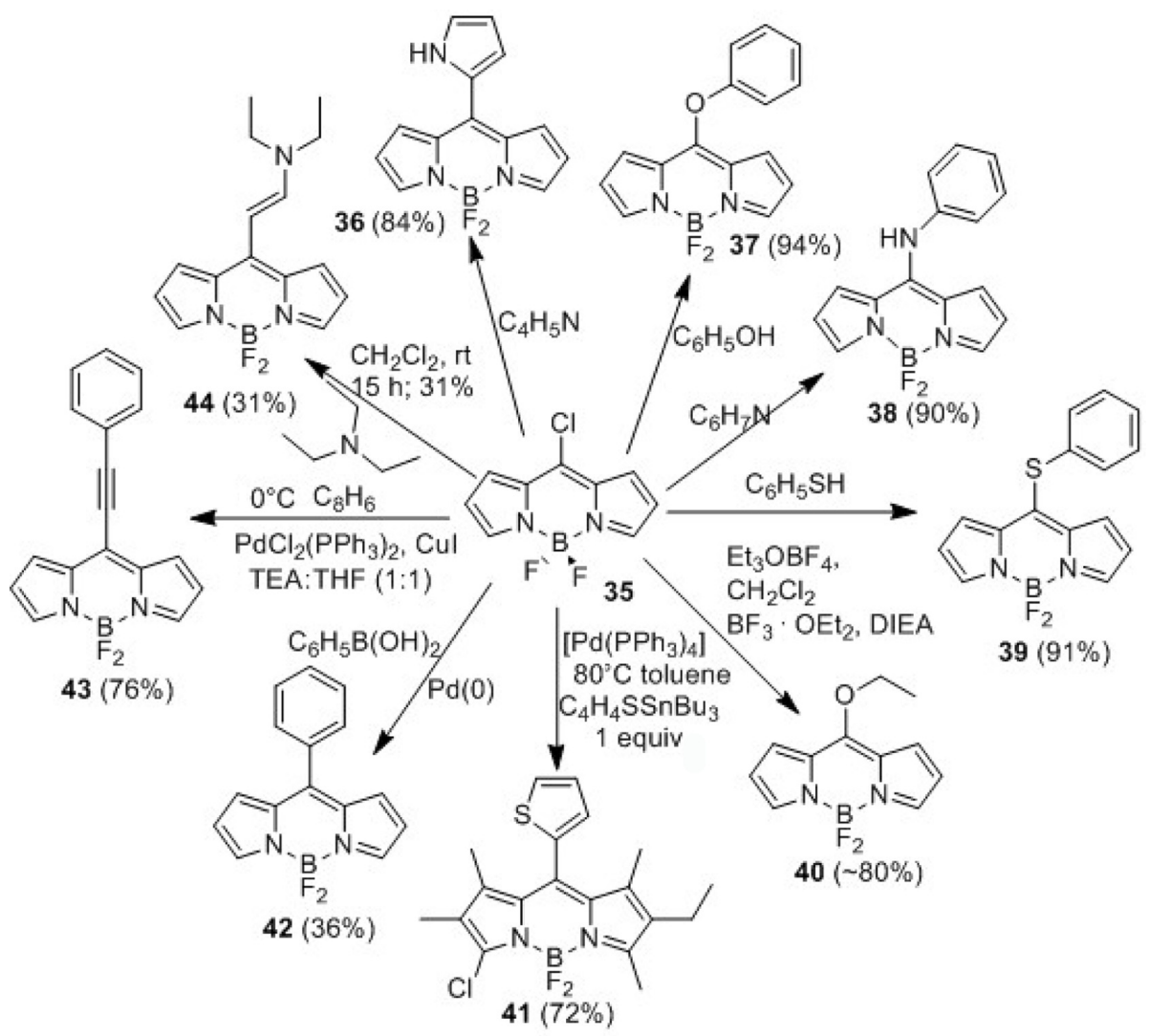

Figure 8 Synthesis of various meso-substituted BODIPY derivatives 36-44 from meso-chloro BODIPY 35.

Abbreviations: BODIPY, boron-dipyrromethene/BF 2 -dipyrrin; h, hours; equiv, equivalent(s); THF, tetrahydrofuran; $r t$, room temperature.

types of aryl, heteroaromatic and ethynyl aromatic groups at the meso-position of BODIPY under $\operatorname{Pd}(0)$ catalyzed Suzuki, Stille, and Sonogashira coupling reactions. They have also showed that meso-chloro BODIPY 35 is a very good synthon for the preparation of meso-aryl, meso-alkyl, and meso-vinyl substituted BODIPYs $41-43$ in high yields using $\operatorname{Pd}(0)$-catalyzed Stille cross-coupling reactions. ${ }^{50-56}$ Misra et al also synthesized a series of meso-arylethynyl BODIPYs 43 by reacting meso-chloro BODIPY 35 with various arylethynes (Figure 8) under Pd-catalyzed Sonogashira cross-coupling reaction and studied the effect of the donor on the photophysical properties of the BODIPYs..$^{50-52,54,56}$ The same group also introduced ${ }^{57}$ enamines at the meso-position of the BODIPY core by coupling 8-chloro BODIPY with tertiary amines and synthesized 44 via catalyst free oxidation of tertiary amines (Figure 8).

\section{Pyrrole functionalized BODIPYs}

The six-pyrrole carbons of the BODIPY core is prone to functionalization and BODIPYs containing one to six functional groups were synthesized and used effectively to prepare the desired substituted BODIPYs. There are different strategies available to prepare pyrrole functionalized BODIPYs.

\section{Mono-functionalized BODIPYs}

The functional group can be introduced either at 2- or 3-position of BODIPY to synthesize 2-functionalized and 3-functionalized BODIPYs.

\section{2-functionalized BODIPYs}

The functional groups such as halogens, formyl, cyano etc, were introduced at the 2-position of BODIPY by various synthetic routes. The halogens such as bromo, chloro, and iodo functional groups were introduced at the 2-position of BODIPY by two different synthetic routes as outlined in Figure 9..$^{58-60} \mathrm{The}$ condensation of 4-halogenated-2-acylpyrrole with the desired substituted pyrrole in the presence of one equivalent of $\mathrm{POCl}_{3}$ resulted in the formation of halogen substituted dipyrromethene which was then neutralized by adding excess of triethylamine followed by complexation with $\mathrm{BF}_{3} \cdot \mathrm{OEt}_{2}$ to afford 2-halogenated BODIPYs 45-47 (Figure 9A). ${ }^{58}$ During this method, the halogen atom was varied from chlorine to bromine to iodine and produced all three mono-halogenated BODIPYs 45-47 in $36 \%-57 \%$ yields. ${ }^{58}$ Alternately, 2-halogenated BODIPYs 45 and 46 were prepared by treating BODIPY 2 with one or less than one equivalent of $\mathrm{N}$-halosuccinimide (NXS) in $\mathrm{CH}_{2} \mathrm{Cl}_{2}$ in high yields since the 2-position of BODIPY 2 is more 

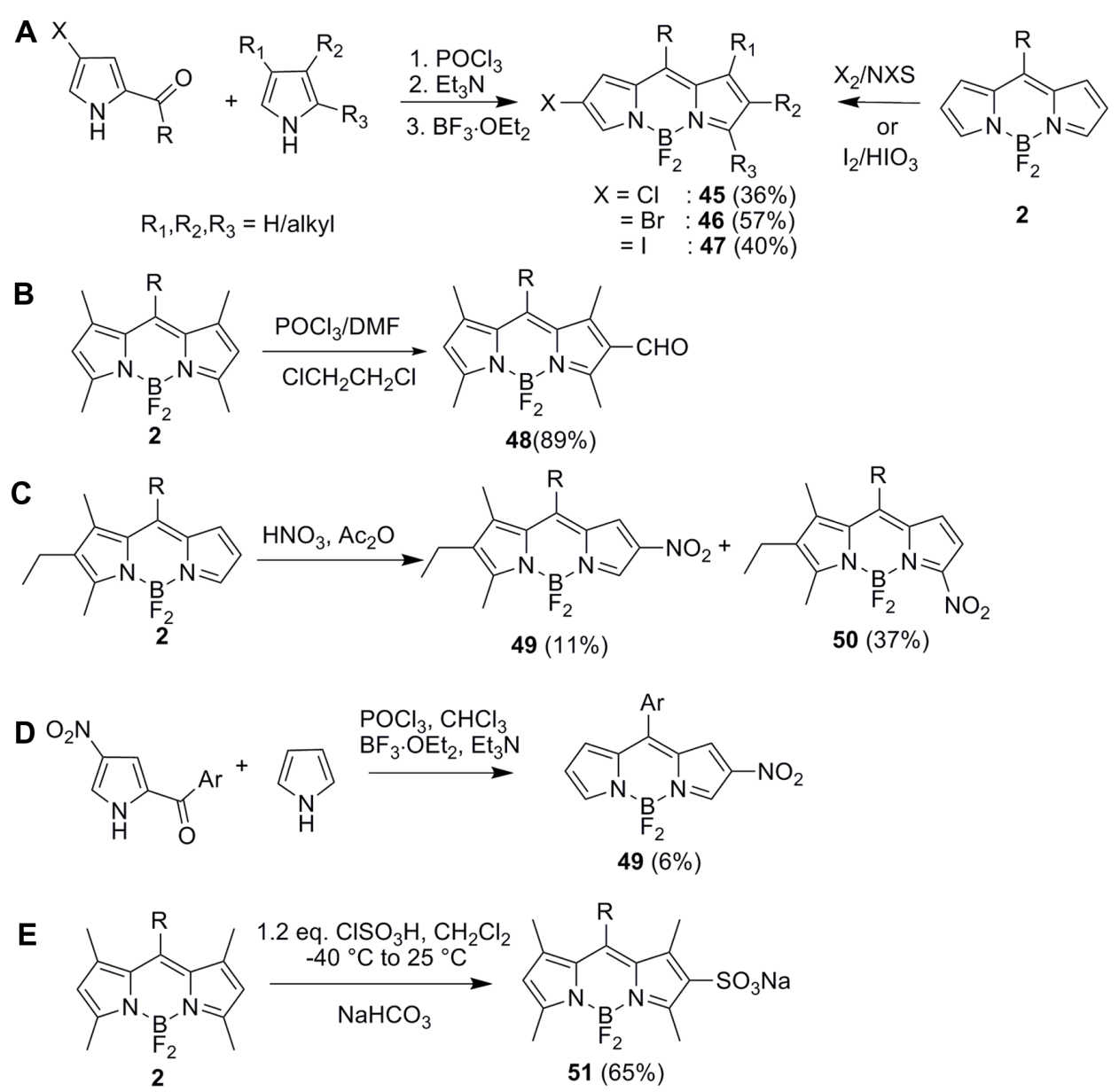

Figure 9 Synthesis of (A) 2-halo, (B) 2-formyl, (C) and (D) 2-nitro and (E) 2-sulfonyl functionalized BODIPYs 45-5I. Abbreviations: BODIPYs, boron-dipyrromethenes/BF 2 -dipyrrins; NXS, N-halosuccinimide; eq, equivalent(s).

prone to electrophilic substitution reactions (Figure 9A). . $^{59,60}$ The halogenation at 2-position of BODIPY 2 was also carried out by treating BODIPY 2 with a brominating agent ${ }^{61}$ such as $\mathrm{Br}_{2}$ and iodinating agent ${ }^{62}$ such as $\mathrm{I}_{2} / \mathrm{HIO}_{3}$ and afforded respective 2-halogenated BODIPYs 46 and 47 in high yields (Figure 8A).

The 2-formylated BODIPY 48 was prepared under modified Vilsmeier-Haack reaction conditions by treating BODIPY 2 with Vilsmeier reagent under mild reaction conditions (Figure 9B) ${ }^{63}$ Recently, Esnal et al attempted to synthesize $\beta$-nitro substituted BODIPY 49 by treating BODIPY 2 with two equivalents of $\mathrm{HNO}_{3}$ and $\mathrm{Ac}_{2} \mathrm{O}$ at $0^{\circ} \mathrm{C}$ (Figure 9C). ${ }^{64}$ This reaction resulted in the formation of a mixture of 5-nitro BODIPY 50 as a major product and 6-nitro BODIPY 49 as a side product. To improve the percentage yield of these mono-substituted BODIPYs 49/50, the authors attempted the nitration reaction by treating nitro-substituted pyrrole with unsubstituted pyrrole under $\mathrm{POCl}_{3}$ acid catalyzed conditions and followed by complexation with $\mathrm{BF}_{3} \cdot \mathrm{OEt}_{2}$ (Figure 9D). ${ }^{64}$
However, the yields of mono-nitro-substituted BODIPY 49 remained the same in both synthetic strategies. The monosulfonyl BODIPY 51 was synthesized by drop-wise addition of 1.2 equivalents of chlorosulfonic acid to the solution of BODIPY 2 (Figure 9E) by maintaining the temperature from $-40^{\circ} \mathrm{C}$ to $25^{\circ} \mathrm{C}$ followed by quenching with $\mathrm{NaHCO}_{3}{ }^{65}$

2-Halo BODIPYs 45-47 were used to prepare various BODIPY derivatives under $\mathrm{Pd}(0)$ cross-coupling conditions. The reaction of 2-bromo BODIPY 46 with bis(pinacolato) diboron $\left(\operatorname{pin}_{2} \mathrm{~B}_{2}\right)$ in the presence of $\mathrm{Cs}_{2} \mathrm{CO}_{3}$ produces $\beta-\beta$ linked BODIPY dimer 52. ${ }^{66}$ The compounds, 2-tetraphenylethene BODIPY 53, 2-ethynylterpyridyl BODIPY 54, and 2-ethynylbipyridyl BODIPY 55 were also synthesized (Figure 10A) under $\operatorname{Pd}(0)$ conditions by the reaction of 2-halo BODIPY with boronic acid/ethynyl compound. ${ }^{62,67,68}$

Using the 2-formyl BODIPY 48, a few BODIPY derivatives were synthesized under Schiff base and Knoevenagel reaction conditions and these derivatives were used for sensing various metal ions. For example, compound oxime BODIPY 56 was 


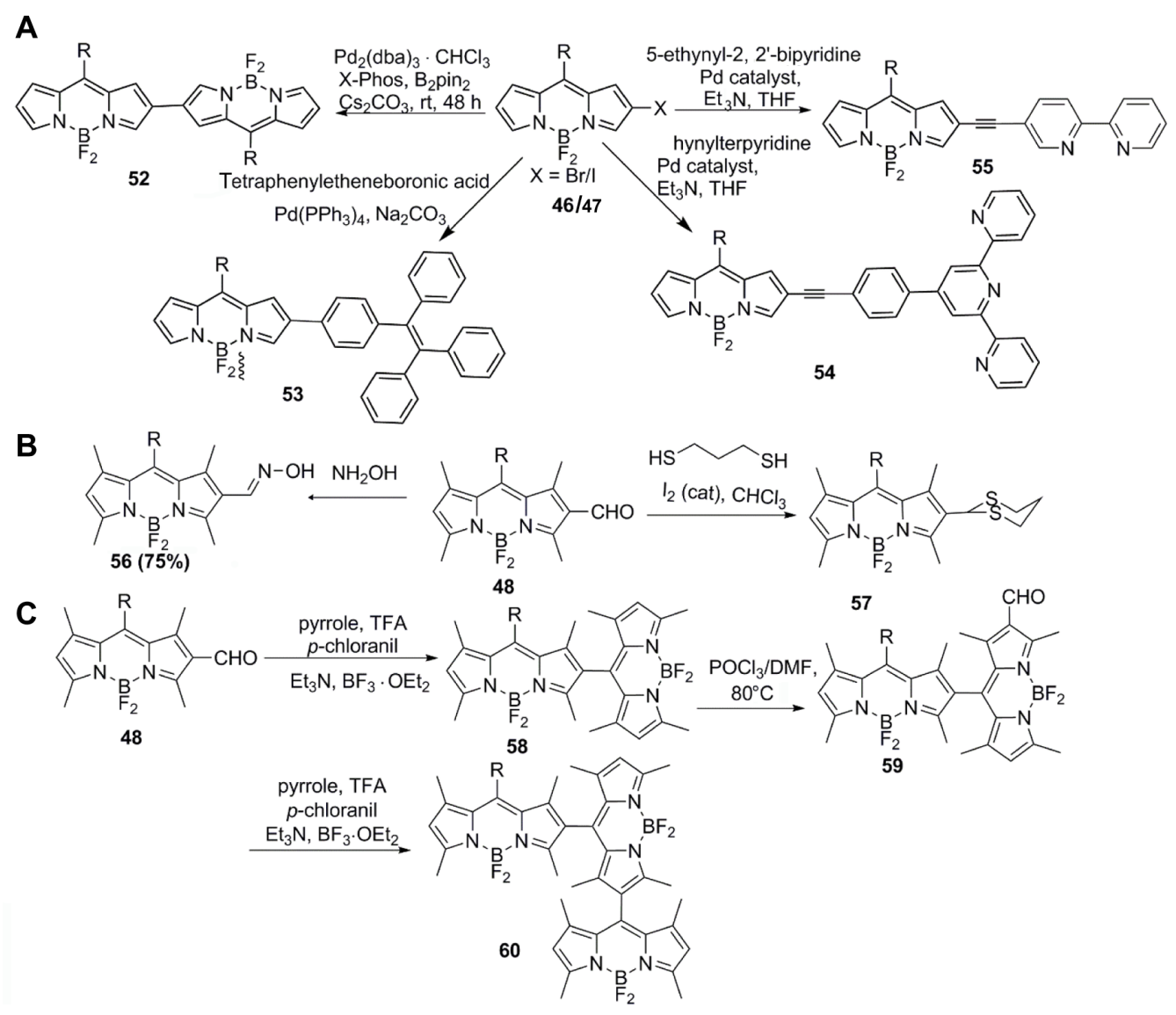

Figure 10 Synthesis of 2-substituted BODIPY derivatives 52-60 derived from (A) 2-halo, (B) and (C) 2-fromyl functionalized BODIPYs. Abbreviations: BODIPYs, boron-dipyrromethenes/BF ${ }_{2}$-dipyrrins; $h$, hours; THF, tetrahydrofuran; TFA, triflouroacetic acid; rt, room temperature.

synthesized by treating 2-formyl BODIPY 48 with hydroxylamine in ethanol at room temperature (Figure 10B) and used to sense $\mathrm{OCl}^{-}$ion whereas compound 57 was used for detection of $\mathrm{Hg}^{2+}$ ion. ${ }^{69,70}$ BODIPY trimer 60 was synthesized from 2-formyl BODIPY 48 in a few steps which involve acid catalytic conditions followed by complexation with $\mathrm{BF}_{3} \cdot \mathrm{OEt}_{2}$ (Figure 10C) to obtain BODIPY dimer 58. The repetition of the same steps under similar conditions on BODIPY dimer 58 yielded the BODIPY trimer 60 in decent yield. ${ }^{71}$

\section{3-functionalized BODIPYs}

The 3-halogenated BODIPYs 61 and 62 were prepared by condensing 5-halogenated 2-acylpyrrole with substituted pyrrole in the presence of $\mathrm{POCl}_{3}$ followed by treatment with excess of $\mathrm{NEt}_{3} / \mathrm{BF}_{3} \cdot \mathrm{OEt}_{2}$ (Figure 11A). ${ }^{58}$ In this method, one can use any substituted pyrrole as second pyrrole to prepare a range of 3-halogenated BODIPYs 61 and 62 . Alternately, treatment of meso-aryl dipyrromethane 1 with one equivalent of appropriate NXS at $-78^{\circ} \mathrm{C}$ in THF followed by oxidation with DDQ and complexation with $\mathrm{BF}_{3} \cdot \mathrm{OEt}_{2}$ in the presence of $\mathrm{Et}_{3} \mathrm{~N}$ (Figure 11A) afforded 3-halogenated
BODIPYs 61 and 62 in $43 \%$ and 33\% yields, respectively. ${ }^{72}$ The 3-halogenated BODIPYs 61 and 62 cannot be prepared by direct substitution of BODIPYs with halogens or any other halogenating reagents. Haefele et al reported the synthesis of 3-formylated BODIPY 63 by DDQ oxidation of 3-methyl BODIPYs 2 (Figure 11B). ${ }^{73}$ The amine group was introduced on the BODIPY core in a few steps. In the first step, the trialkyl substituted pyrrole was treated with sodium aqueous nitrite and acetic acid/acetic anhydride (Figure 11C) to afford 3-amine substituted dipyrromethene $64 .{ }^{74}$ However, during this step, some acylated dipyrrin 65 was also formed. In the subsequent step, the mixture of dipyrromethenes complexed with $\mathrm{BF}_{3} \cdot \mathrm{OEt}_{2}$ in the presence of triethylamine and the resulted crude mixture of BODIPYs were purified by column chromatography to afford 3-amino substituted BODIPY 66 in decent yield. The 3-amino BODIPY 66 was also synthesized by reducing the corresponding nitro BODIPY 50 in the presence of molecular hydrogen (Figure 11D) under $\mathrm{Pd} / \mathrm{C}$ catalyzed conditions. ${ }^{64}$ Alternately, the 3 -amino BODIPY 66 was also synthesized from 3-bromo BODIPY 62 in three steps. In the first step, the 3-bromo BODIPY 62 was treated with 
A
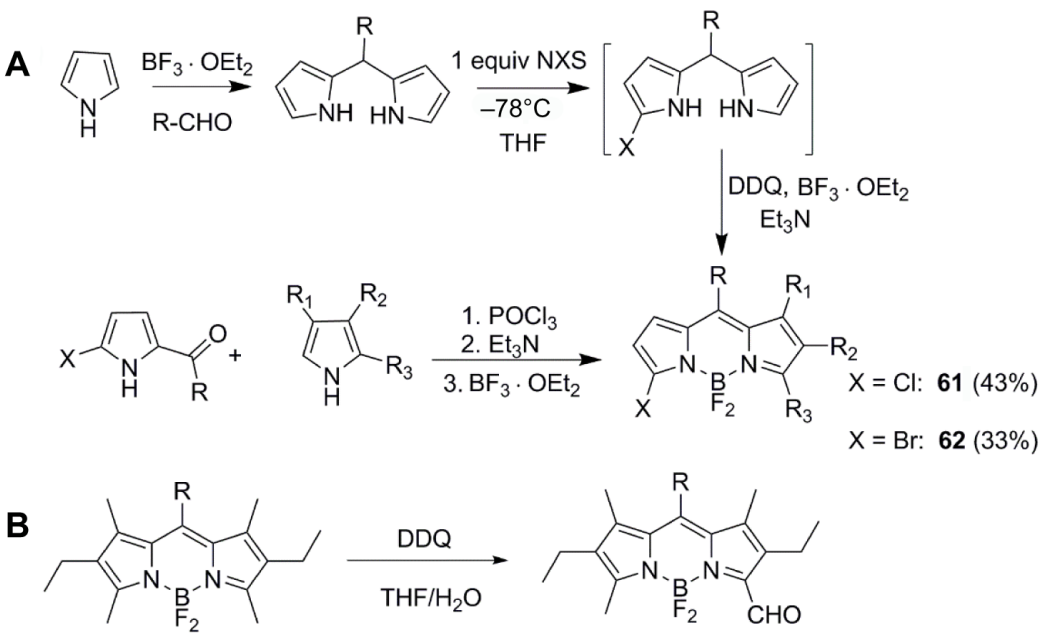

2

$63(50 \%)$
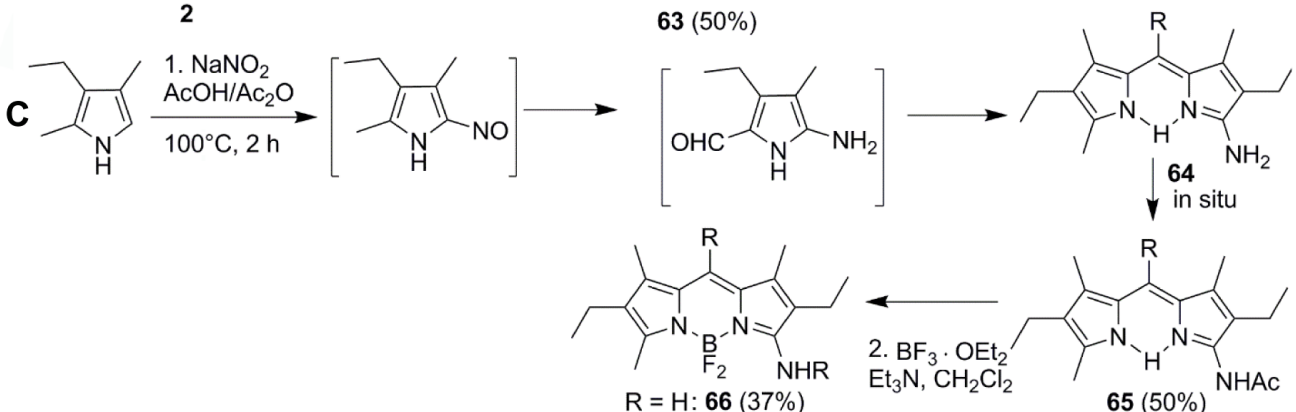

$\mathrm{R}=\mathrm{H}: \mathbf{6} \mathbf{6}(37 \%)$

$65(50 \%)$

D

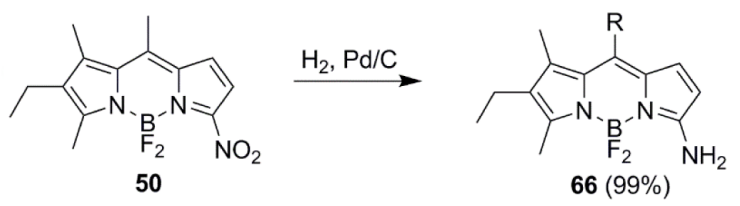

E

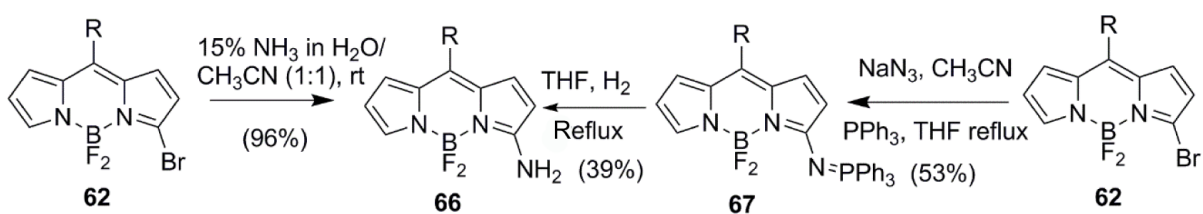

Figure I I Synthesis of (A) 3-halo, (B) 3-formyl, (C-E) 3-amino functionalized BODIPYs 6I-66.

Abbreviations: BODIPYs, boron-dipyrromethenes/BF 2 -dipyrrins; h, hours; equiv, equivalent(s); NXS, N-halosuccinimide; THF, tetrahydrofuran; rt, room temperature; DDQ, 2,3-dichloro-5,6-dicyano-I,4-benzoquinone.

sodium azide in acetonitrile to generate 3-azido BODIPY which was then reacted with $\mathrm{PPh}_{3}$ in THF (Figure 11E) and afforded 3-iminophosphorane BODIPY 67. In the subsequent step, 3-iminophosphorane BODIPY 67 was refluxed in THF/ water and yielded 3-amino BODIPY $66 .^{75}$

3-Halo BODIPYs 61 and 62 were subjected to Pd(0) crosscoupling conditions and synthesized a variety of BODIPY derivatives using Suzuki, Stille, and Sonogashira methods. The reaction of 3-bromo BODIPY 62 with ethynylanthracene under Sonoagashira $\mathrm{Pd}(0)$ cross-coupling conditions (Figure 12A) produced ethyne bridged BODIPY-anthracene conjugate 68 , which showed efficient energy transfer from anthracene to BODIPY unit. ${ }^{72}$ The 3-mono-halogenated BODIPYs 61 and 62 were also subjected to nucleophilic substitution reactions
(Figure 12B) by treating with different types of nucleophiles in the presence of base at reflux conditions. The reaction of 3-bromo BODIPY 62 with 3-hydroxypyridine produced 3-oxypyridine BODIPY 69 which was further used to prepare non-covalent BODIPY-Zn(II) porphyrin dyad 70 and BODIPY-Ru(II) porphyrin dyad 71 (Figure 12B) assembled via $\mathrm{Zn}(\mathrm{II}) / \mathrm{Ru}(\mathrm{II})$-pyridine "N" interaction. ${ }^{76}$ The 3-amino BODIPY 66 was used to prepare 2,4-dinitrobenzenesulfonyl (DBS) appended BODIPY 72 by treating with DBS chloride (Figure 12C) in the presence of NaH. ${ }^{77}$ The BODIPY 72 is non-fluorescent due to photo-induced electron transfer from BODIPY to DBS group. However, in the presence of thiophenols which cleave the DBS group, the fluorescence is restored and thus 72 can be used as turn-on fluorescent probe 


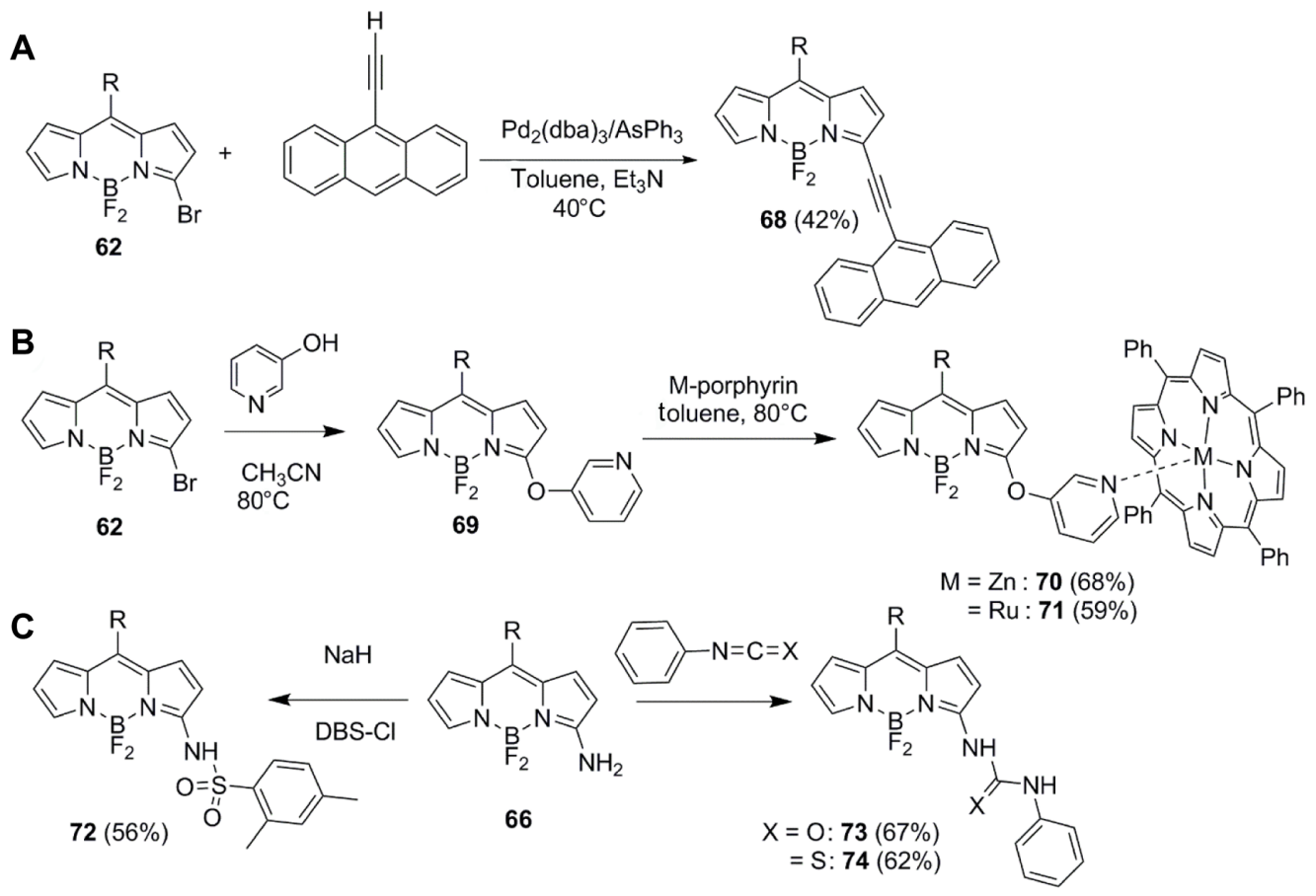

Figure 12 Synthesis of 3-substituted BODIPY derivatives 68-74 derived from (A) 3-bromo, (B) 3-formyl and (C) 3-amino functionalized BODIPYs.

Abbreviations: BODIPYs, boron-dipyrromethenes/BF 2 -dipyrrins; DBS, 2,4-dinitrobenzenesulfonyl.

for selective detection of thiophenols. ${ }^{77}$ The BODIPY urea/ thiourea derivatives 73 and 74 were prepared (Figure 12C) by the reaction of 3-amino BODIPY 66 and phenylisocyanate and phenylisothiocyanate respectively under $\mathrm{CH}_{3} \mathrm{CN}$ reflux conditions. ${ }^{75}$ The phenyl urea BODIPY 73 acts as colorimetric and ratiometric sensor for $\mathrm{F}^{-}$ion and thiourea BODIPY 74 can act as specific chemodosimetric sensor for $\mathrm{Hg}^{2+}$ ion. ${ }^{75}$

\section{Difunctionalized BODIPYs}

There are several reports on difunctionalized BODIPYs. Since there are six possible positions on the BODIPY core where the two functional groups can be introduced selectively to prepare difunctionalized BODIPYs, the various difunctionalized BODIPYs were prepared as described here.

\section{2,6-difunctionalized BODIPYs}

The 2,6-positions of BODIPY 2 bear the least positive charge and are susceptible to electrophilic attack. The 2,6dihalogenated BODIPYs 75-77 were prepared by treating appropriate BODIPY with NXS or $\mathrm{X}_{2}\left(\mathrm{Br}_{2}, \mathrm{Cl}_{2}, \mathrm{I}_{2}\right)$ in $\mathrm{CH}_{2} \mathrm{Cl}_{2}$ (Figure 13A) at room temperature or other slightly varied reaction conditions. ${ }^{61,62}$ Recently Wang et al showed that the regioselective dihalogenation at 2,6-positions of BODIPY 2 can be much faster when reactions were carried out in hexafluoro-2-propanol (Figure 13A) using NXS as the halogen source. ${ }^{78}$ The 2,6-diformyl BODIPY 78 was prepared by treating 2-formyl BODIPY 48 under Vilsmeier-Haack reaction conditions (Figure 13B).$^{79}$ However, recently it was shown that the 2,6-diformyl BODIPY 78 can be prepared in one pot by treating BODIPY 2 under Vilsmeier-Haack reaction conditions by in situ generation of 2-formyl BODIPY 48 (Figure 13C). ${ }^{80}$ The 2,6-dinitro BODIPY 79 was also synthesized (Figure 13D) by treating BODIPY 2 with nitric acid at $0^{\circ} \mathrm{C}$. ${ }^{81}$ Similarly 2,6-dicyano BODIPY 81 was synthesized from 2,6-diformyl BODIPY 78 in two steps. In the first step, the 2,6-dioxime BODIPY 80 was prepared by treating 2,6-diformyl BODIPY 78 with $\mathrm{NH}_{2} \mathrm{OH}$ in ethanol and the resulting 2,6-dioxime BODIPY 80 was treated in a second step with $(\mathrm{COCl})_{2}$ in $\mathrm{CH}_{3} \mathrm{CN}$ (Figure 13E) to afford 2,6dicyano BODIPY $81 .{ }^{80}$ Alternately, the 2,6-dicyano BODIPY 81 was also synthesized by reacting 3-cyano-2,4-dimethyl substituted pyrrole with acetoxyacetyl chloride followed by complexation with $\mathrm{BF}_{3} \cdot \mathrm{OEt}_{2}$ (Figure $13 \mathrm{~F}$ ) in the presence of diisopropylethylamine. ${ }^{82}$ The 2,6-disulfonyl BODIPY 83 (Figure 13G) was prepared by using two equivalents of chlorosulfonic acid followed by quenching with $\mathrm{NaHCO}_{3}{ }^{65}$

The donor-acceptor-donor system 84 was synthesized by coupling 2,6-dihalogenated BODIPY 76 with 4-borat-4,4' dimethyl triphenylamine under transition metal catalyzed reaction conditions (Figure 14A). ${ }^{83}$ The donor-acceptordonor type triphenylamine substituted BODIPY 84 used in bulk heterojunction solar cells as electron donor exhibited photovoltaic properties with a power conversion efficiency of $0.77 \%$. The 2,6-bis(ethynylterpyridyl) BODIPY 85 was 

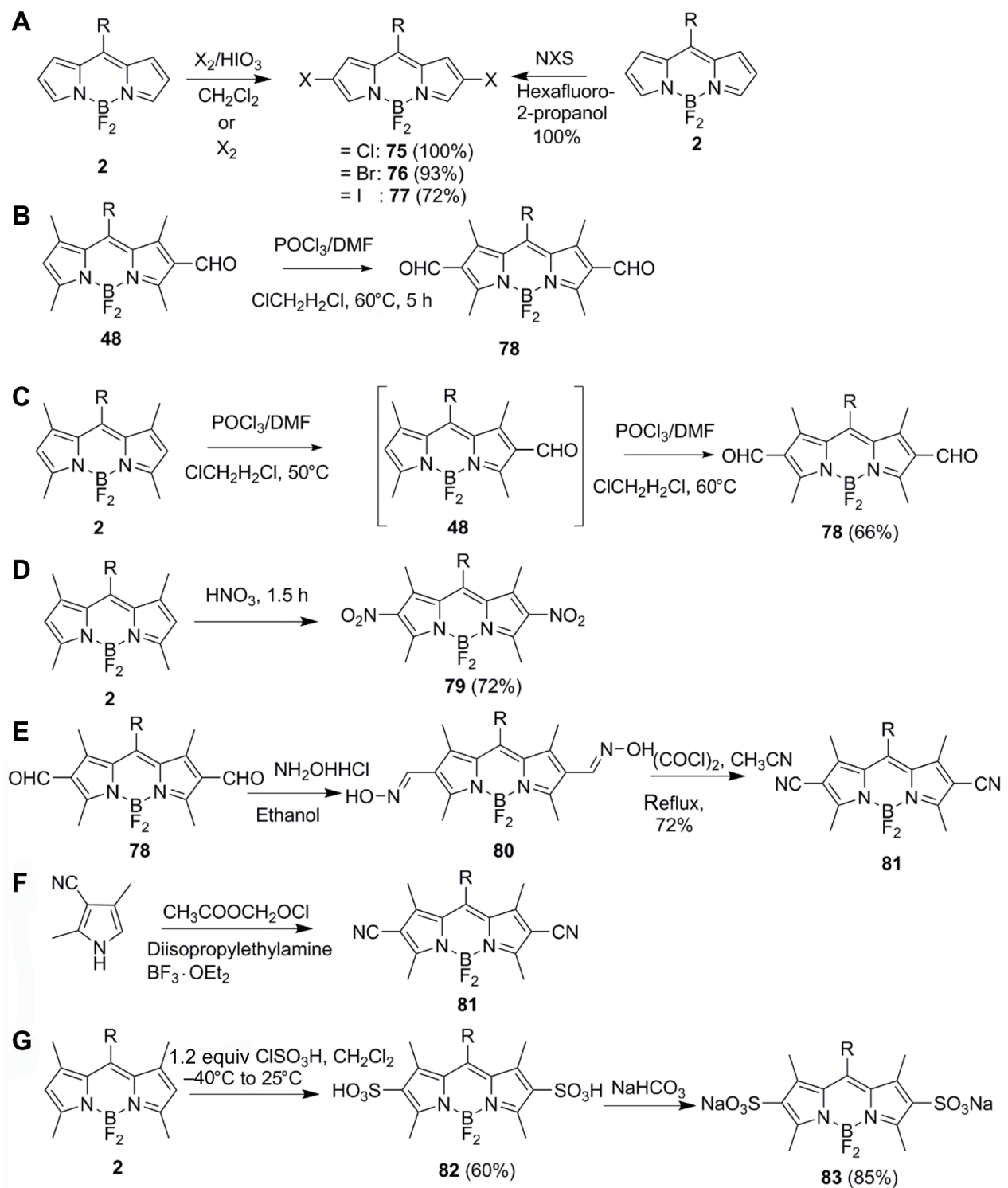

Figure 13 Synthesis of (A) 2,6-dihalo, (B) and (C) 2,6-diformyl, (D) 2,6-dinitro, (E) and (F) 2,6-dicyano and (G) 2,6-disulfonyl functionalized BODIPYs 75-83. Abbreviations: BODIPYs, boron-dipyrromethenes/BF 2 -dipyrrins; NXS, N-halosuccinimide; h, hours; equiv, equivalent(s).

prepared (Figure 14B) by the reaction of 2,6-dihalo BODIPY 77 with ethynylterpyridyl under Sonogashira coupling conditions. ${ }^{62}$ The formyl groups present at 2,6-pyrrole carbons of BODIPY 78 were used mainly for Schiff base and Knoevenagel condensation reactions and produced new functionalized BODIPYs 86-92 as shown in Figure 14C. The 2,6-dihydroxyl BODIPY 92 was prepared by reducing the formyl groups two steps; the 2,6-dicarboxylic acid substituted BODIPY 91 was prepared by oxidation of formyl groups of 2,6-diformyl BODIPY 78 with $\mathrm{NaClO}_{2}$; the hydroxypropyl substituted BODIPY 88 was synthesized by treating 2,6-diformyl BODIPY 78 with ethylmagnesium bromide; and the dialkenylnitro substituted BODIPY 90, divinylcyanoacetic acid substituted BODIPY 87 were synthesized under Knoevenagel conditions by treating the 2,6-diformyl BODIPY 78 with nitromethane/cyanoacetic acid.$^{80}$ The dialkenylester substituted BODIPY 89 was synthesized under Wittig reaction conditions by treating 2,6-diformyl BODIPY 78 with phosphorous ylide (Figure 14C). ${ }^{80}$ All these $\pi$-extended conjugated BODIPYs exhibited a $40-50 \mathrm{~nm}$ bathochromic shift in their absorption and emission bands compared to 2,6-diformyl BODIPY 78 with moderate Stokes shifts and low quantum yields.

\section{3,5-difunctionalized BODIPYs}

The 3,5-dihalogenated BODIPYs 93 and 94 were prepared starting with meso-aryl dipyrromethane 1 as shown in Figure 15A. Treatment of meso-aryl dipyrromethane 1 with two equivalents of appropriate NXS in THF at $-78^{\circ} \mathrm{C}$ followed by oxidation with DDQ and complexation with 

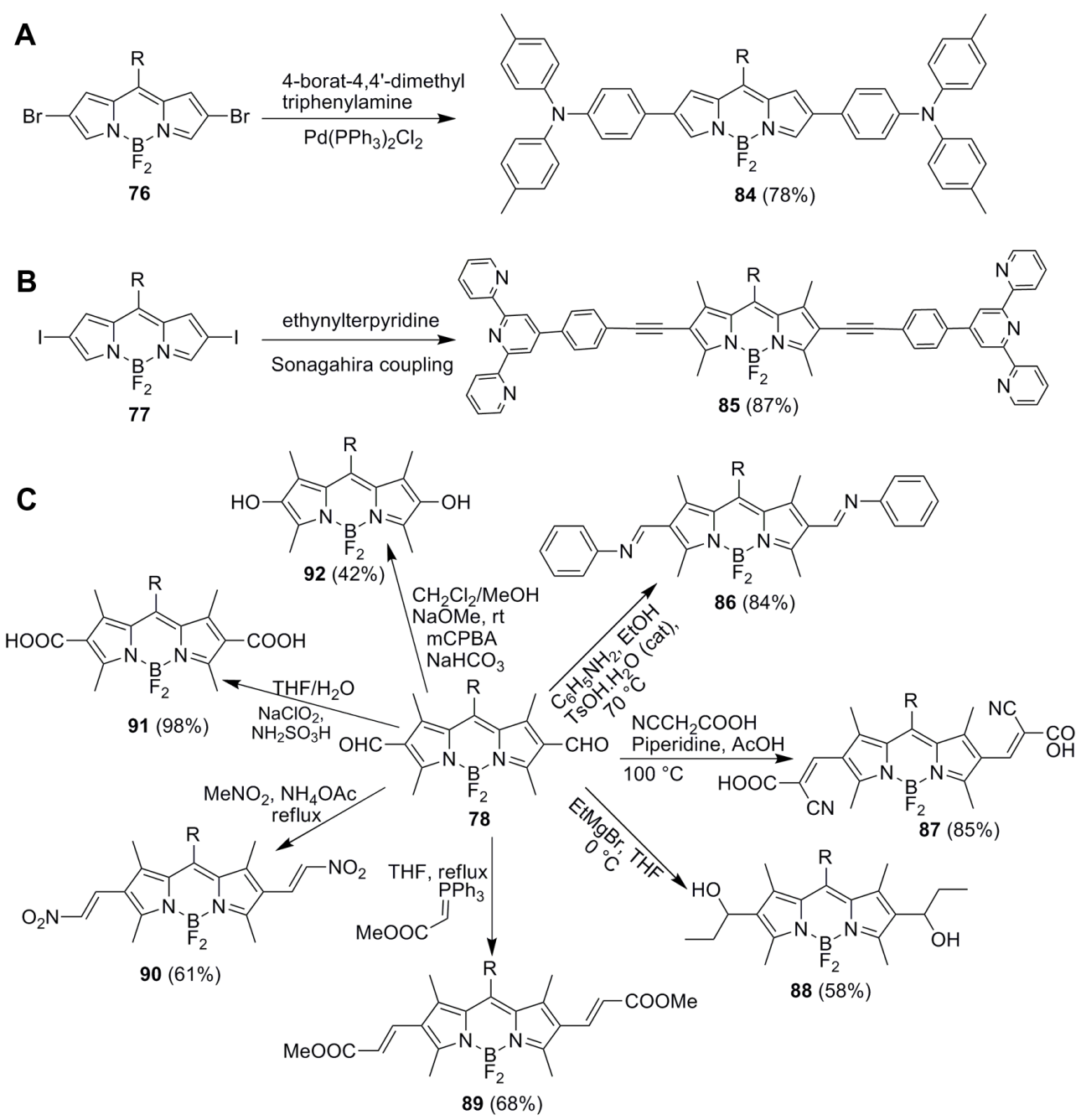

Figure 14 Synthesis of 2,6-disubstituted BODIPYs 84-92 from (A) 2,6-dibromo, (B) 2,6-diiodo and (C) 2.6-diformyl functionalized BODIPYs. Abbreviations: BODIPYs, boron-dipyrromethenes/ $\mathrm{BF}_{2}$-dipyrrins; $\mathrm{THF}$, tetrahydrofuran; $r$, room temperature; cat, catalyst.

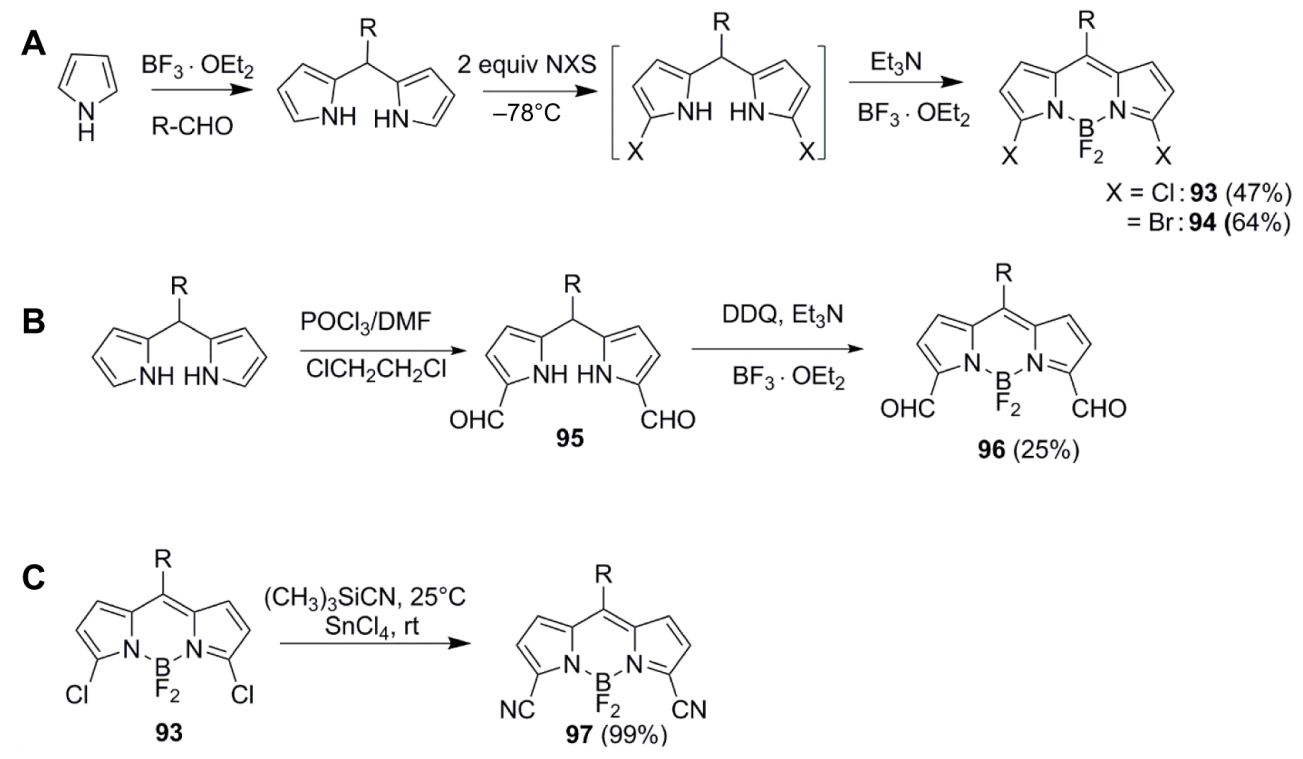

Figure 15 Synthesis of (A) 3,5-dihalo, (B) 3,5-diformyl and (C) 3,5-dicyano functionalized BODIPYs 93-97.

Abbreviations: BODIPYs, boron-dipyrromethenes/BF -dipyrrins; equiv, equivalent(s); NXS, N-halosuccinimide; DDQ, 2,3-dichloro-5,6-dicyano-I,4-benzoquinone; rt, room temperature. 

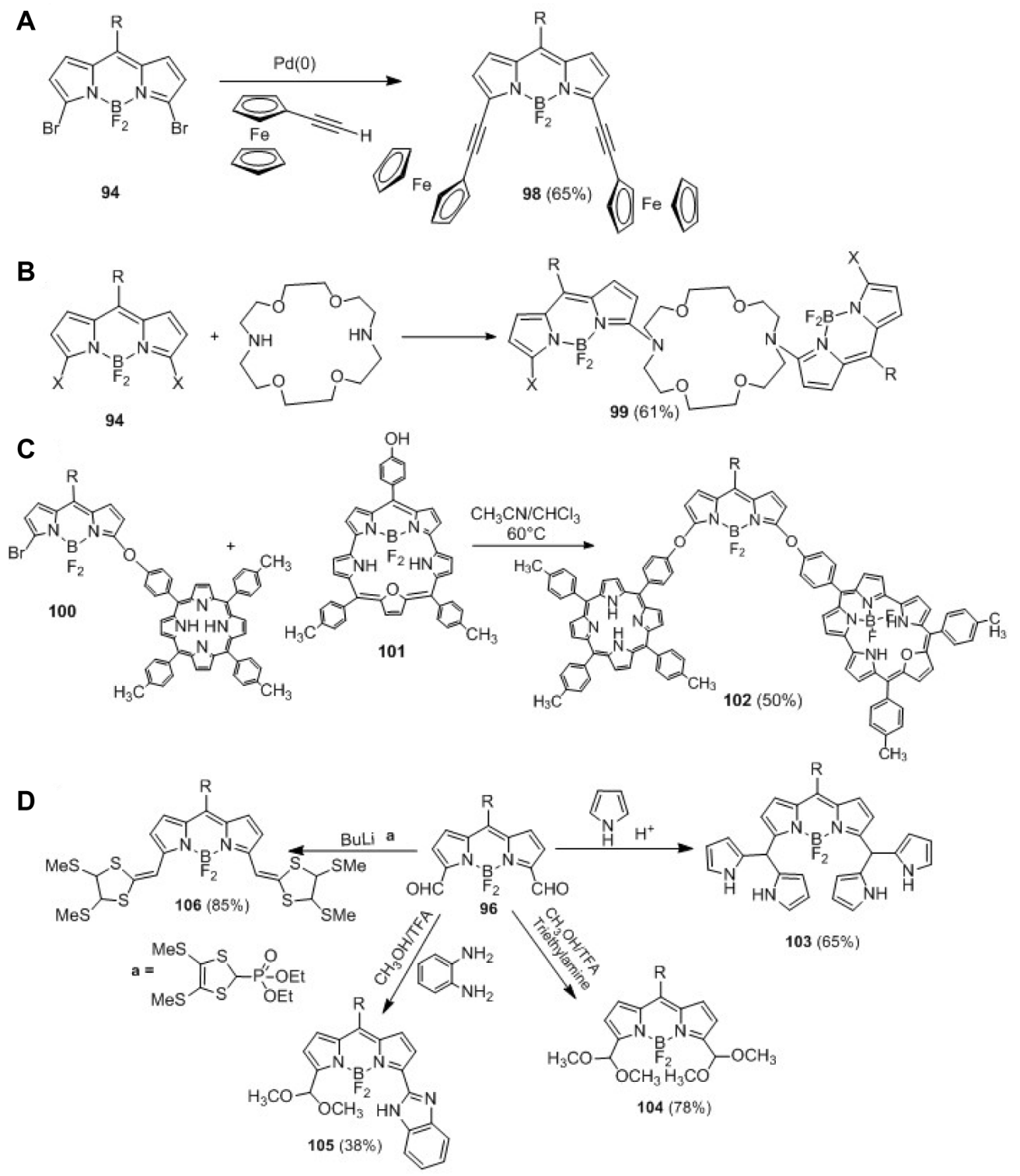

Figure 16 Synthesis of 3,5-disubstituted BODIPY derivatives 98-106 derived from 3,5-dihalo (A-C), and 3,5-diformyl (D) functionalized BODIPYs. Abbreviation: BODIPY, boron-dipyrromethene/BF ${ }_{2}$-dipyrrin.

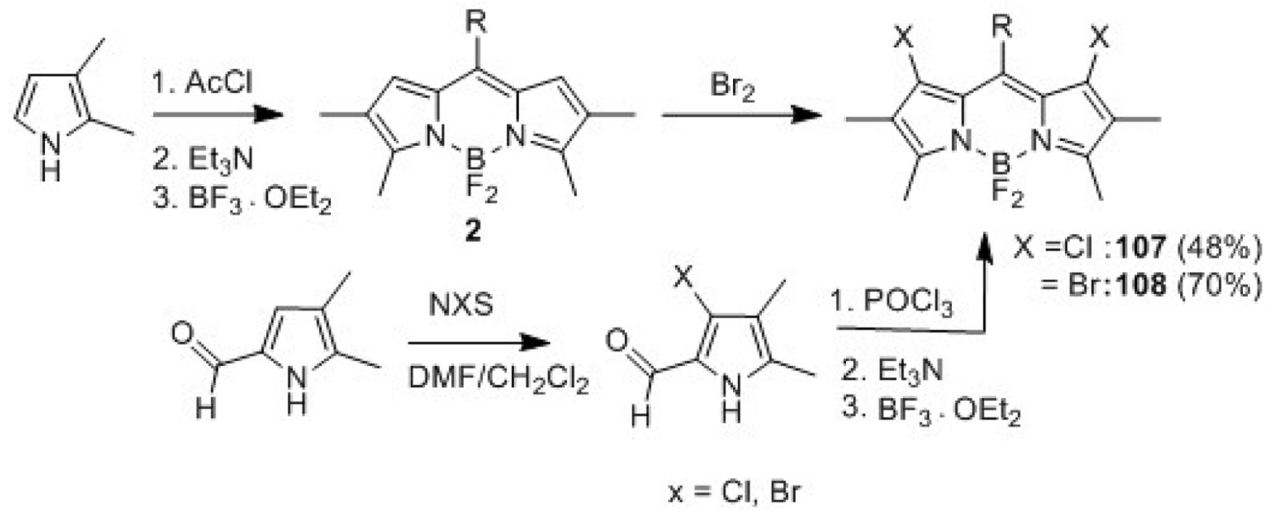

Figure 17 Synthesis of I,7-dihalogenated BODIPYs 107 and 108.

Abbreviations: BODIPYs, boron-dipyrromethenes/BF ${ }_{2}$-dipyrrins; $\mathrm{NXS}, \mathrm{N}$-halosuccinimide. 

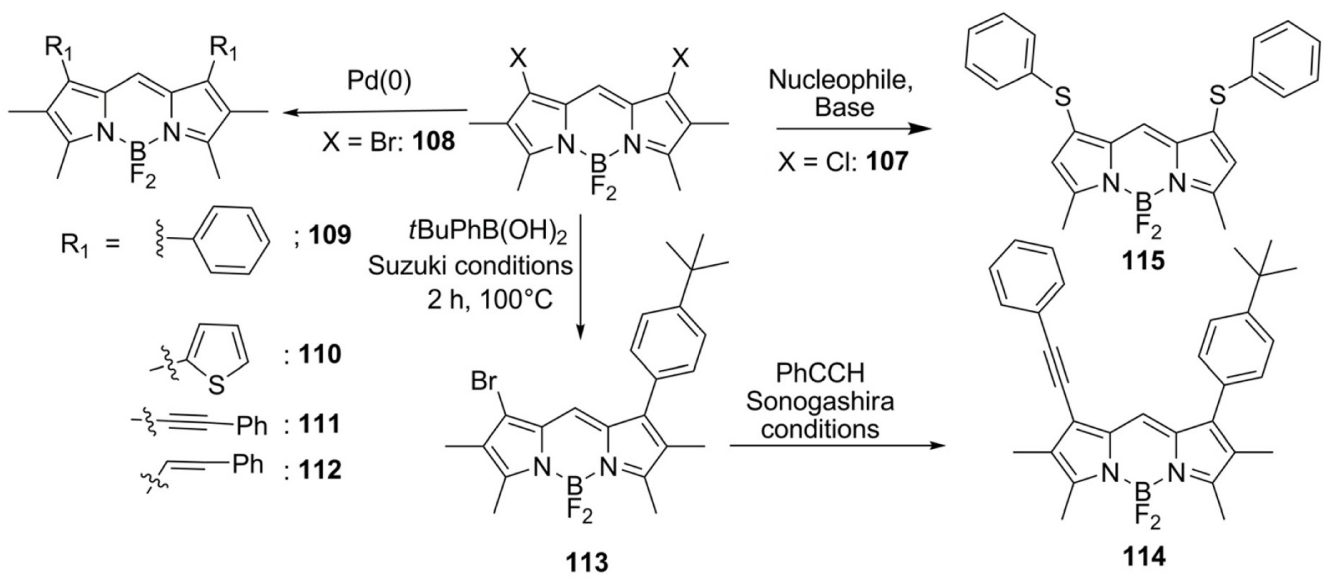

Figure 18 Synthesis of I,7-disubstituted BODIPY derivatives 109-II5.

Abbreviations: BODIPY, boron-dipyrromethene/ $\mathrm{BF}_{2}$-dipyrrin; h, hours.

$\mathrm{NEt}_{3} / \mathrm{BF}_{3} \cdot \mathrm{OEt}_{2}$ afforded the 3,5-dihalogenated BODIPYs 93 and $94 .{ }^{84-86}$ The other halogenated BODIPYs were also formed in these reactions but column chromatography was used to separate the 3,5-dihalogenated BODIPYs 93 and 94 from the mixture of halogenated BODIPYs. This is the only reliable method available to synthesize 3,5-dihalogenated BODIPYs 93 and 94. The meso-aryl substituted 3,5-diformyl BODIPY 96 was prepared in decent yield by oxidizing the 1,9-diformyl dipyrromethane 95 with DDQ, followed by treatment with triethylamine and complexation with $\mathrm{BF}_{3} \cdot \mathrm{OEt}_{2}$ (Figure 15B). ${ }^{87}$ Later on, Yu et al also reported the 3,5-diformyl-meso-anisyl BODIPY by following the same reaction conditions. ${ }^{88}$ The 3,5-dicyano BODIPY 97 was synthesized by treating 3,5dichloro BODIPY 93 with trimethylsilyl cyanide and $\mathrm{SnCl}_{4}$ (Figure 15C) at room temperature. ${ }^{89}$

Different types of symmetrically or asymmetrically aryl/ alkyl substituted BODIPY derivatives were synthesized from 3,5-dihalo BODIPYs 93/94 by the Stille, Suzuki, Heck and Sonogashira coupling reactions. The accessibility of 3,5-dihalo BODIPYs 93/94 helped in the preparation of a wide range of 3,5-diaryl substituted BODIPYs under various Pd catalyzed coupling conditions in good yields. For example, 3,5-diethynylferrocene BODIPY 98 was synthesized
(Figure 16A) from the reaction of 3,5-dibromo BODIPY 94 with ethynylferrocene under $\operatorname{Pd}(0)$ Sonogashira coupling conditions. ${ }^{85}$ The presence of conjugated aryl groups at the 3,5-positions alters the electronic properties of the dye significantly, which was reflected in their spectral and electrochemical properties. ${ }^{7}$ Furthermore, the 3,5-dihalo BODIPYs 93 and 94 were also used for various nucleophilic substitution reactions by treating with $\mathrm{C}-, \mathrm{O}-, \mathrm{N}-, \mathrm{S}-, \mathrm{Se}-$, and $\mathrm{Te}-$ nucleophilic groups at reflux conditions (Figure 16B). ${ }^{86}$ The BODIPYs containing two different types of porphyrinoids at 3,5-positions were also synthesized (Figure 16C) by treating 3-bromo-5-porphyrinyl BODIPY 100 with 4-hydroxyphenyl$\mathrm{BF}_{2}$-oxasmaragdyrin 101 in $\mathrm{CHCl}_{3}$ at $60^{\circ} \mathrm{C} .9^{90}$

The 3,5-diformyl BODIPYs (96) are brightly fluorescent in solution state and exhibit good optical properties. ${ }^{87}$ The 3,5-diformyl BODIPYs (96) were used as fluorescent $\mathrm{pH}$ sensors $^{87}$ and also as exclusive chemodosimetric and colorimetric sensors for $\mathrm{CN}^{-}$ion detection. ${ }^{91}$ The 3,5-bis(dipyrromethanyl) BODIPY 103 was synthesized by treating the 3,5-diformyl BODIPY 96 with pyrrole under acidic conditions (Figure 16D). ${ }^{92}$ The bis(dipyrromethanyl BODIPY) 103 showed a specific selectivity for fluoride ion due to the presence of four $\mathrm{NH}$ groups that have the ability to bind $\mathrm{F}^{-}$ion strongly. ${ }^{92}$ The

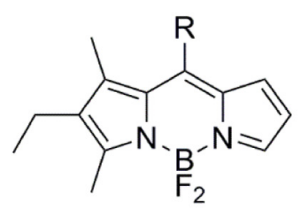

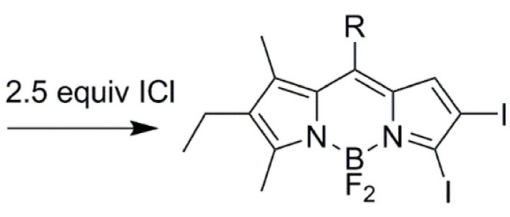

$116(67 \%)$

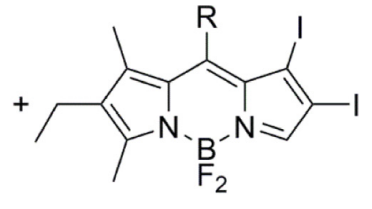

$117(10 \%)$

Figure 19 Synthesis of I,2/2,3-diiodo BODIPYs II6 and II7

Abbreviations: BODIPYs, boron-dipyrromethenes/BF ${ }_{2}$-dipyrrins; equiv, equivalent(s). 

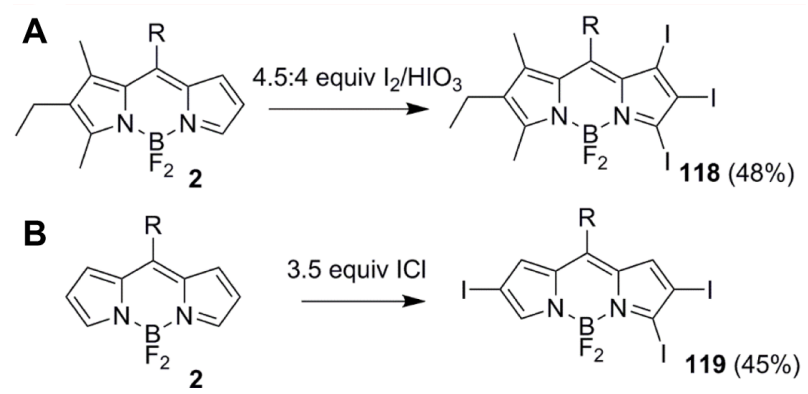

Figure 20 Synthesis of (A) I,2,3- triiodo BODIPY II8 and (B) 2,3,6-triiodo BODIPY 119.

Abbreviations: BODIPYs, boron-dipyrromethenes/BF 2 -dipyrrins; equiv, equivalent(s).

BODIPY containing acetal groups at 3,5-position 104 was synthesized by treating 3,5-diformyl BODIPY 96 with a catalytic amount of TFA (trifluoroacetic acid) in methanol at room temperature and demonstrated as a fluorescent chemodosimeter for the detection of explosive picric acid in aqueous medium (Figure 16D).$^{93}$ The benzimidazole containing BODIPY derivative 105 was prepared by treating 3,5-diformyl BODIPY 96 with o-phenylenediamine in $\mathrm{CH}_{3} \mathrm{OH}$ in the presence of a catalytic amount of trifluoroacetic acid (Figure 16D) and demonstrated its use as reversible and reusable selective chemosensor for fluoride detection. ${ }^{94}$ Recently Bill et al reported a "on-off-on" electrochromic system, the $\pi$-extended tetrathiafulvalene substituted BODIPY 106 synthesized by treating 3,5-diformyl BODIPY 96 with phosphonate ester under Horner-WadsworthEmmons type coupling conditions. ${ }^{95}$

\section{I,7-difunctionalized BODIPYs}

The 1,7-positions of BODIPY 2 are electron poor like 3,5positions and direct halogenation on BODIPY 2 at these positions is not possible if other positions are not blocked with the substituents. Leen et al synthesized the first examples of 1,7-dihalogenated BODIPYs 107 and 108 by adopting two synthetic routes shown in Figure $17 .{ }^{96}$ In the first strategy, the authors condensed the 2,3-dimethylpyrrole with acetyl chloride in $\mathrm{CH}_{2} \mathrm{Cl}_{2}$ followed by in situ complex formation to afford 2,3,5,6-tetrasubstituted BODIPY, which was subjected to bromination with $\mathrm{Br}_{2}$ in $\mathrm{CH}_{2} \mathrm{Cl}_{2}$ and afforded 1,7-dibrominated BODIPY 108 in 82\% yield. In the second strategy, the 2,3-dimethylpyrrole was first formylated at 5-position under standard Vilsmeier-Haack reaction conditions, which was then halogenated at 4-position by treating with NXS and afforded 2,3-dimethyl-4-halo-5-formyl pyrrole. ${ }^{96}$ The substituted pyrrole was then treated with phosphorus oxychloride to afford dipyrromethene that was then complexed with $\mathrm{BF}_{3} \cdot \mathrm{OEt}_{2}$ and obtained 1,7-dihalogenated BODIPYs 107 and 108 as sole product (Figure 17). Thus, the 1,7-dihalogenated BODIPYs 107 and 108 can be prepared by only blocking all other pyrrolic positions of BODIPY. ${ }^{96}$

The 1,7-dihalo substituted BODIPYs 107 and 108 were subjected to $\operatorname{Pd}(0)$ cross-coupling reactions such as Suzuki, Heck, and Sonogashira coupling reactions 109-114 as well as nucleophilic substitution reactions (Figure 18). ${ }^{96}$ Among the nucleophiles, only sulfur based nucleophiles were introduced at 1,7-positions of BODIPY 115. The extension of conjugation at 1,7-positions of BODIPY did not alter the optical properties significantly compared to 3,5-positions of BODIPY. ${ }^{96}$

\section{I,2/2,3-difunctionalized BODIPYs}

Oritz et al reported synthesis of 2,3- diiodinenated 116 and 1,2-diiodinenated 117 BODIPYs by treating BODIPY 2 with 2.5 equivalents of $\mathrm{ICl}$ in $\mathrm{CH}_{2} \mathrm{Cl}_{2} / \mathrm{CH}_{3} \mathrm{OH}$ and the resulting mixtures of diiodenenated BODIPYs 116 and 117 were separated by column chromatography (Figure 19)..$^{97}$ These kinds of dihalogenated BODIPYs 116 and 117 can be prepared only by blocking the second pyrrole ring carbons of BODIPY unit 2.

\section{Trifunctionalized BODIPYs}

Jiao et al reported regiospecific bromination of BODIPYs by treating BODIPY 2 with liquid bromine but they failed
A<smiles></smiles>

2

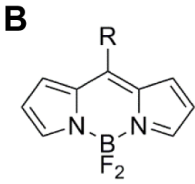

2

B
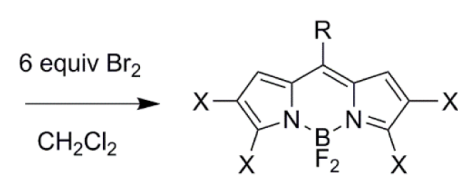

$120(95 \%)$

Figure 2 I Synthesis of $(\mathbf{A})$ tetrabromo and $(\mathbf{B})$ tetrahalogenated BODIPYs 120-123. Abbreviations: BODIPYs, boron-dipyrromethenes/ $\mathrm{BF}_{2}$-dipyrrins; equiv, equivalent(s). 


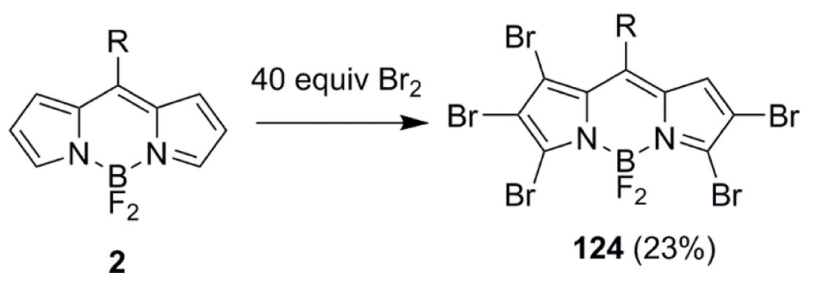

Figure 22 Synthesis of pentahalogenated BODIPY 124.

Abbreviations: BODIPY, boron-dipyrromethene/BF 2 -dipyrrin; equiv, equivalent(s).

to isolate pure tribromo BODIPY. ${ }^{61}$ Oritz et al prepared the 1,2,3-trihalogenated BODIPY 118 by taking BODIPY 2 in which one of the pyrrole ring carbons were blocked and treated with 4.5:4 ratio of $\mathrm{I}_{2} / \mathrm{HIO}_{3}$ in $\mathrm{CH}_{2} \mathrm{Cl}_{2} / \mathrm{CH}_{3} \mathrm{OH}$ at room temperature (Figure 20A). ${ }^{97}$ The reaction resulted in the formation of a mixture of two different types of BODIPYs such as 1,2,3-trihalogenated BODIPY 118 and 2,3-dihalogenated BODIPY which were separated by column chromatography. The same research group also reported the synthesis (Figure 20B) of 2,3,6-trihalogenated BODIPY 119 by treating meso-aryl BODIPY 2 with 3.5 equivalents of $\mathrm{ICl}$ and the resulting mixtures of halogenated BODIPYs were separated by column chromatography to afford 2,3,6-trihalogenated BODIPY 119. ${ }^{97}$

\section{Tetrafunctionalized BODIPYs}

Jiao et al reported the synthesis of 2,3,5,6-tetrabromo BODIPY 120 by treating BODIPY 2 with 6 equivalents of $\mathrm{Br}_{2}$ in $\mathrm{CH}_{2} \mathrm{Cl}_{2}$ (Figure 21A). ${ }^{61}$ This method afforded the desired tetrabrominated BODIPY 120 along with other brominated BODIPYs and were separated by column chromatography. Oritz et al prepared tetraiodinated BODIPY 121 and other tetrahalogenated BODIPYs with two different types of halogens such as iodo and chloro groups 122 and 123 by treating
BODIPY 2 with 4.5 equivalents of $\mathrm{ICl}$ in $\mathrm{CH}_{2} \mathrm{Cl}_{2} / \mathrm{CH}_{3} \mathrm{OH}$ (Figure 21B) and isolated the tetrahalogenated BODIPYs 121-123 by column chromatography. ${ }^{97}$

\section{Pentafunctionalized BODIPYs}

Jiao et al prepared pentahalogenated BODIPY 124 by treating BODIPY 2 with 40 equivalents of $\mathrm{Br}_{2}$ in $\mathrm{CH}_{2} \mathrm{Cl}_{2}$ (Figure 22). ${ }^{61}$ In this strategy, the pentahalogenated BODIPY 124 was formed as major product along with other minor brominated BODIPYs and column chromatography was used to separate and isolate the pentahalogenated BODIPY 124 from other halogenated BODIPYs.

\section{Hexafunctionalized BODIPYs}

Jiao et al attempted to prepare hexabrominated meso-aryl BODIPY 125 by treating meso-aryl BODIPY 2 with 300 equivalents of $\mathrm{Br}_{2}$ in $\mathrm{CH}_{2} \mathrm{Cl}_{2}$ (Figure 23A) and obtained a decent yield. ${ }^{61}$ 1,2,3,5,6,7-Hexahalogenated BODIPYs 125 and 126 were also prepared by treating meso-aryl dipyrromethane 1 with excess equivalents of NXS in THF at room temperature followed by oxidation with DDQ and complexation with $\mathrm{BF}_{3} \cdot \mathrm{OEt}_{2}$ (Figure 23B). ${ }^{59,98}$

The tri, tetra, and pentahalogenated BODIPYs were used to synthesize corresponding aryl substituted BODIPYs 128-130 by reacting with appropriate arylboronic acids at $80^{\circ} \mathrm{C}$ (Figure 24A) under Suzuki coupling conditions..$^{99}$ The nucleophilic substitution of tetrachloro BODIPY 136 with pyrrole under reflux conditions produced 2,6-dichloro-3, 5-dipyrrolyl BODIPY 127 (Figure 24A). ${ }^{100}$ Furthermore, the hexahalogenated BODIPY 125 was used extensively to prepare a variety of sterically crowded hexasubstituted BODIPYs 131 and 132 such as hexaphenyl, hexatolyl, hexafluorophenyl, hexa-anisyl and hexanaphthyl. ${ }^{101}$

A

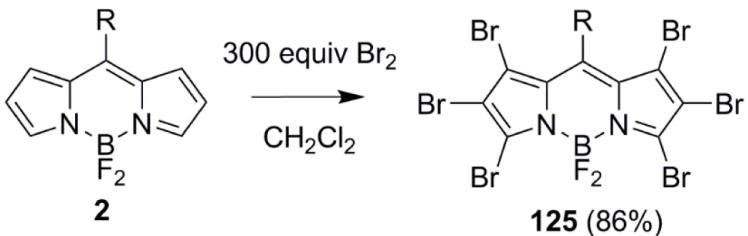

B

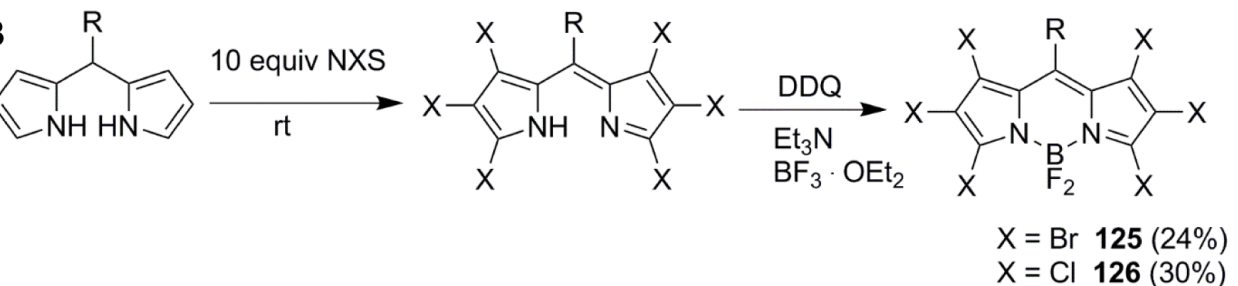

Figure 23 (A and B) Synthesis of hexahalogenated BODIPYs I25 and I 26.

Abbreviations: BODIPYs, boron-dipyrromethenes/BF ${ }_{2}$-dipyrrins; equiv, equivalent(s); NXS, N-halosuccinimide; DDQ, 2,3-dichloro-5,6-dicyano-I,4-benzoquinone; rt, room temperature. 

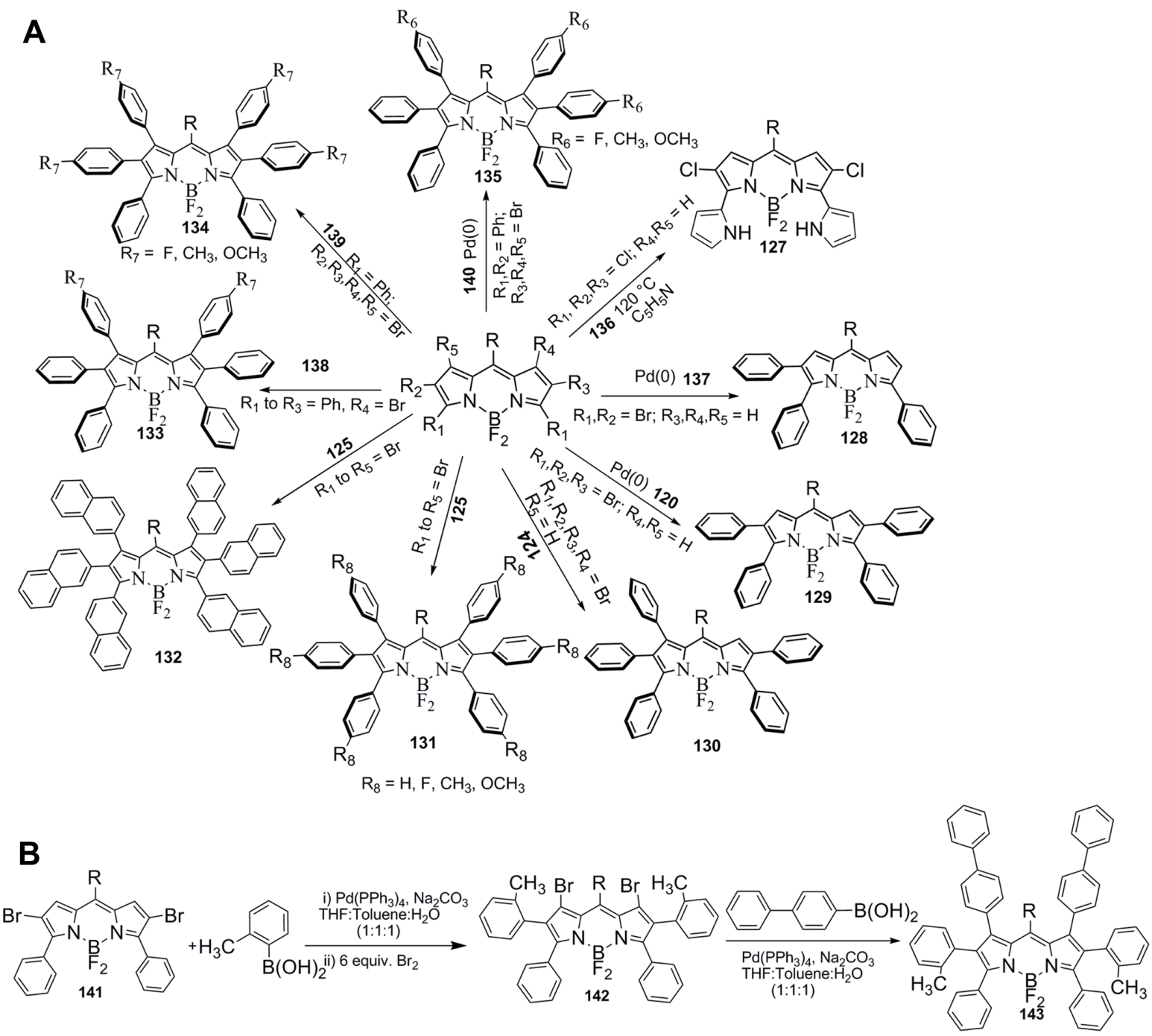

Figure 24 Synthesis of (A) tri, tetra, and various types of polyarylated BODIPYs I27-I35 and (B) hexaarylated BODIPY having three different types of substituents I43. Abbreviations: BODIPYs, boron-dipyrromethenes/BF 2 -dipyrrins; equiv, equivalent(s); THF, tetrahydrofuran.

The hexasubstituted BODIPYs with two different types of substituents (aryl/alkyl) at pyrrole carbons 133-135 were also synthesized in a step-wise manner under $\operatorname{Pd}(0)$ catalyzed Suzuki coupling conditions by coupling the appropriate bromo BODIPY and boronic acid at $80^{\circ} \mathrm{C} .{ }^{102}$ For example, 3,5-diphenyl,1,2,6,7-tetrabromo BODIPY 139 was coupled with different boronic acids such as fluorophenylboronic acid, tolylboronic acid, anisylboronic acid, and methylboronic acid under Suzuki coupling conditions to synthesize (Figure 24A) the corresponding hexasubstituted BODIPY with two different types (aryl/alkyl) of groups 133-135. ${ }^{102}$ Hexasubstituted BODIPY with three different types of aryl groups 143 was synthesized in a sequence of steps which involve several halogenated reactions followed by $\operatorname{Pd}(0)$ catalyzed coupling reactions. ${ }^{103}$ The 1,7-dibromo-3,5-diphenyl-2,6di(o-tolyl) BODIPY 142 was synthesized (Figure 24B) in two steps from 2,6-dibromo-3,5-diphenyl BODIPY 141 by coupling with o-tolylboronic acid followed by bromination with six equivalents of $\mathrm{Br}_{2}$. In the next step, the 1,7-dibromo3,5-diphenyl-2,6-di(o-tolyl) BODIPY 142 was treated with 4-biphenylboronic acid at $80^{\circ} \mathrm{C}$ to afford 1,7-bis(biphenyl)3,5-diphenyl-2,6-di(o-tolyl) BODIPY $143 .{ }^{103}$

\section{Functionalization at the B(II) center of BODIPYs}

In addition to functionalization of the BODIPY core at the pyrrole carbons and meso-aryl group, recently it was realized that even fluorides of $\mathrm{BF}_{2}$ moiety of BODIPY can be substituted under various conditions (Figures 25 and 26). Ulrich et al reported the first substitution of $\mathrm{F}$ atoms with ethynylarenes by treating BODIPY with lithiated ethnylaryls (Figure 25A) in THF under mild reaction conditions. ${ }^{104}$ Using this approach, 


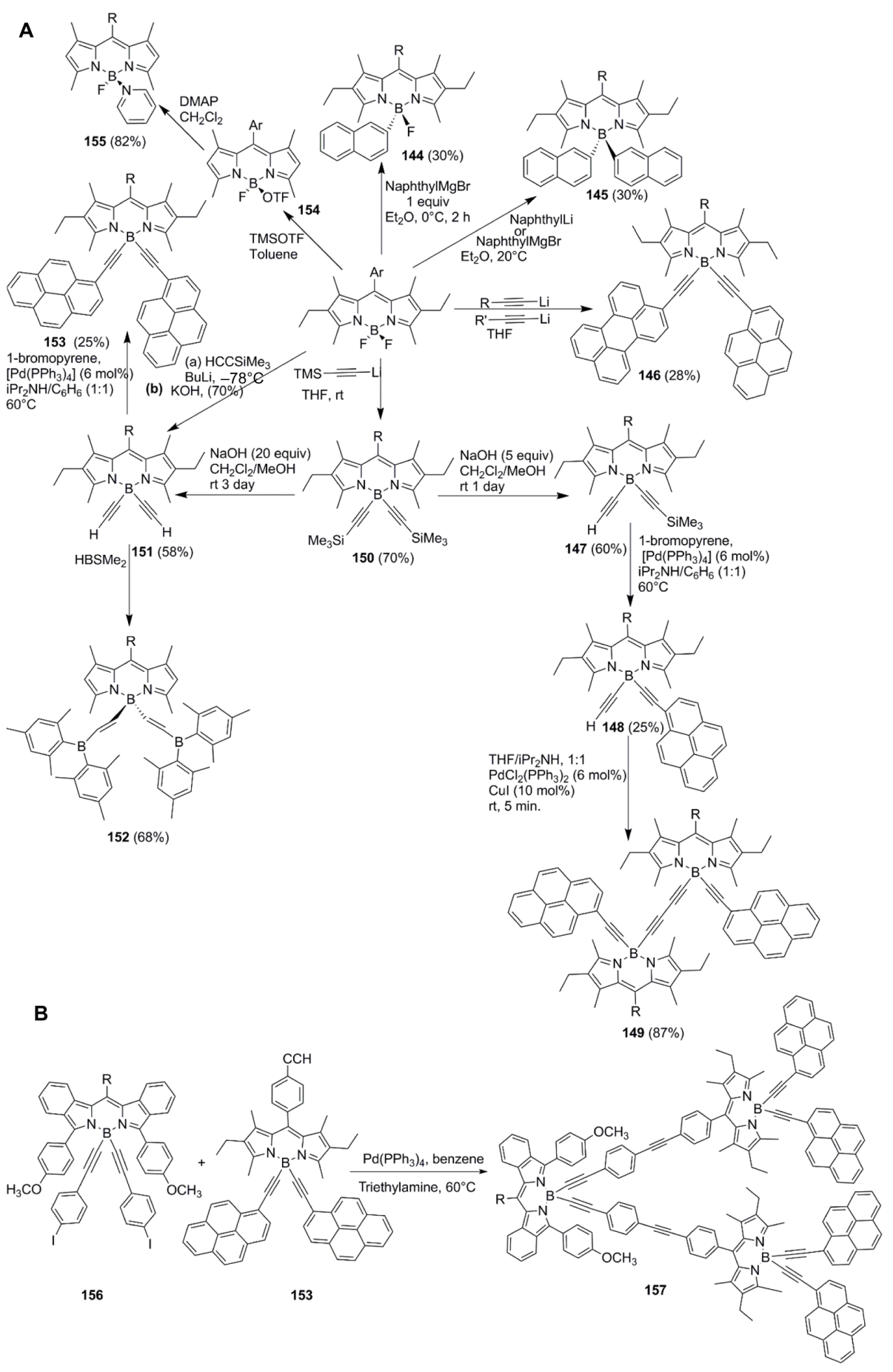

Figure 25 Synthesis of (A) ethynyl substituted and (B) cascade type ethynyl substituted BODIPY-chromophore conjugates I44-157.

Abbreviations: BODIPY, boron-dipyrromethene/BF-dipyrrin; equiv, equivalent(s); $h$, hours; TMSOTF, trimethylsilyl trifluoromethanesulfonate; min, minutes; THF, tetrahydrofuran; rt, room temperature.

they introduced a wide range of ethynylaryl groups at the boron center by replacing the fluoride atoms. Thus, ethynylaryl groups such as ethynylpyrene, ethynylperylene, ethynylanthracene, ethynylterpyridine along with other simple protected ethynyls were introduced (Figure 25A and B) at the boron center and produced several interesting compounds 146-153 and 156 and $157 .{ }^{105}$ In some of these systems, such as ethy- nylpyrene substituted BODIPY 153, highly efficient energy transfer was observed from pyrene to the BODIPY moiety. This synthetic approach also allowed synthesis of asymmetrical derivatives ${ }^{106}$ bearing two different ethnylaryl groups at the boron center such as compound 146-148. Furthermore, Goze et al used their approach more efficiently and prepared a series of donor-spacer-acceptor triads as shown for the preparation 

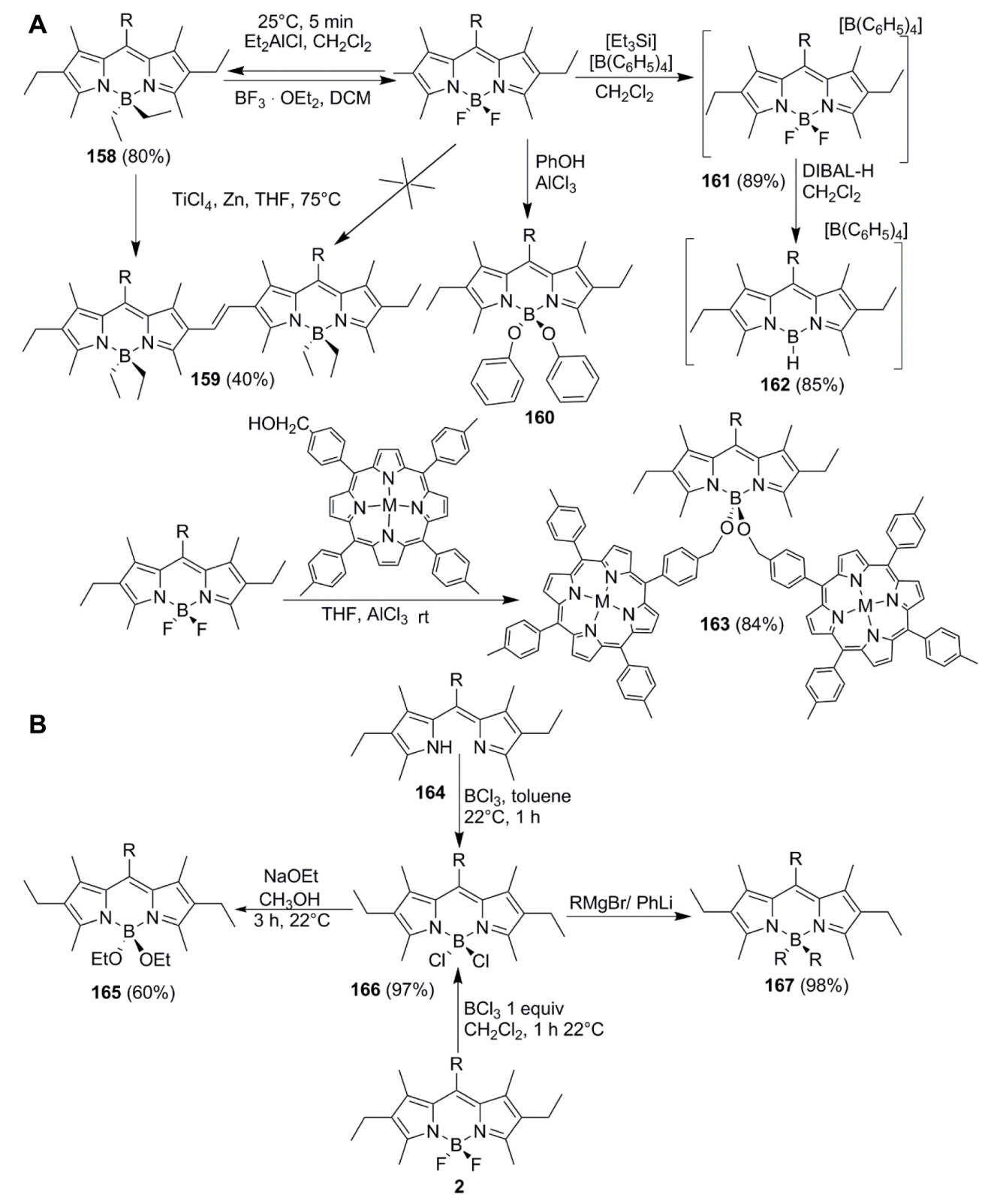

Figure 26 Synthesis of F-substituted BODIPY derivatives I58-167 derived from (A) $\mathrm{BF}_{2}-\mathrm{BODIPY}$ and $(\mathbf{B}) \mathrm{BCl}_{2}-\mathrm{BODIPY}$.

Abbreviations: BODIPY, boron-dipyrromethene/ $\mathrm{BF}_{2}$-dipyrrin; equiv, equivalent(s); h, hours; min, minutes; THF, tetrahydrofuran.

of compound 149 and explored their intramolecular energy transfer properties. ${ }^{107,108}$

Ulrich et al also synthesized a series of various BODIPY bearing substituents such as butyl, phenyl, naphthyl, pyrenyl etc, by treating BODIPY with organometallic reagents (aryl/alkyl lithium and Grignard reagent) under mild conditions (Figure 25A) ${ }^{109}$ These reactions yielded either mono-substituted 144 or disubstituted 145 products depending on reaction conditions. ${ }^{110}$ These compounds were highly fluorescent and exhibited interesting redox properties. $\mathrm{Lu}$ et al reported the synthesis of BODIPY derivatives 152 (Figure 25A) where $\mathrm{F}$ atoms were substituted by $\mathrm{BMeS}_{2}$ (vinyl) groups by treating bis-acetylide BODIPY 151 with $\mathrm{HBSMe}_{2}$ under hydroboration reaction conditions. ${ }^{111}$

Tahtaoui et al synthesized $\mathrm{B}(\mathrm{OR})_{2}$-dipyrrin complexes 160,163 by treating BODIPY with appropriate alkyl/aryl alcohol (Figure 26) in the presence of $\mathrm{AlCl}_{3}{ }^{112,113}$ Hudnall and Gabbai synthesized $p$-dimethylaminopyridine adduct of BODIPY by treating BODIPY with trimethylsilyltriflate followed by dimethylaminopyridine and the resulting adduct 155 was used for the detection of fluoride ions. ${ }^{114}$ Recently, Bonnier et al reported the synthesis ${ }^{115}$ of borenium cations 161 (Figure 26) resulting from the abstraction of fluoride of a BODIPY, by treating it with trimethylsilyl 
ion/pentafluoroarylborate ion and the borenium cation was converted to a borenium hydride 162 by treatment with DIBAL-H. Recently, $\mathrm{BCl}_{2}$-dipyrrin 166 was prepared ${ }^{116,117}$ by treating $\mathrm{BODIPY}$ with one equivalent of $\mathrm{BCl}_{3}$ in $\mathrm{CH}_{2} \mathrm{Cl}_{2}$ or by direct reaction of dipyrrin 164 with $\mathrm{BCl}_{3}$ in toluene at room temperature (Figure 26B). The $\mathrm{BCl}_{2}$-dipyrrin complex was also used to prepare alkyl/aryl substituted B-dipyrrin complex 167 as well as alkyloxy or aryloxy substituted B-dipyrrin complexes 165 under similar reaction conditions used for BODIPYs (Figure 26B). ${ }^{116,117}$ More et al prepared $\mathrm{BEt}_{2}$-dipyrrin $158^{118}$ by treating BODIPY with $\mathrm{Et}_{2} \mathrm{AlCl}$ and also easily converted $\mathrm{BEt}_{2}$-dipyrrin back to BODIPY by reacting with $\mathrm{BF}_{3} \cdot \mathrm{OEt}_{2}$ in $\mathrm{CH}_{2} \mathrm{Cl}_{2}$. This masking and demasking strategy was used to synthesize a BODIPY dimer 159 by McMurry coupling (Figure 26A). Thus, fluoride atoms of $\mathrm{BF}_{2}$ moiety of BODIPY are also susceptible to various reactions and allow for a wide variety of substituted boron dipyrrin complexes.

\section{Conclusion}

Functionalized BODIPYs are highly desirable to prepare a wide variety of substituted BODIPYs that can have potential applications in research fields ranging from materials and biology to medicine. The BODIPYs are synthetically very easily accessible and have several potential sites where functionalization can be carried out to prepare the functionalized BODIPYs. Earlier, the functional group(s) were introduced at the meso-aryl group by following the standard synthetic protocol of condensing the functionalized aryl aldehydes with pyrrole under mild acid catalyzed conditions followed by oxidation and complexation with $\mathrm{BF}_{2}$ group. However, in recent times, several strategies have been developed to introduce a variety of functional groups at different sites on the BODIPY core. Thus, methods were developed to introduce the functional groups directly at the meso-position, at all pyrrole carbons of the BODIPY core, and also at the boron center. These functionalized BODIPYs were further used to prepare the desired complex BODIPYs and explore their applications in different fields, which have been discussed in other recently published reviews. ${ }^{12-16}$ We anticipate that future efforts will be directed toward introducing a larger variety of functional groups on BODIPY including water-soluble groups and using dyes more effectively for various applications.

\section{Acknowledgment}

MR thanks the Department of Science and Technology for funding and RS thanks the University Grants Commission for the fellowship.

\section{Disclosure}

The authors have no conflicts of interest to disclose.

\section{References}

1. Dsouza RN, Pischel U, Nau WM. Fluorescent dyes and their supramolecular host/guest complexes with macrocycles in aqueous solution. Chem Rev. 2011;111(12):7941-7980.

2. Parish CP. Fluorescent dyes for lymphocyte migration and proliferation studies. Immunol Cell Biol. 1999;77(6):499-508.

3. Hawe A, Sutter M, Jiskoot W. Extrinsic fluorescent dyes as tools for protein characterization. Pharm Res. 2008;25(7):1487-1499.

4. Ragab SS, Swaminathan S, Bakerb JD, Raymo FM. Activation of BODIPY fluorescence by the photoinduced dealkylation of a pyridinium quencher. Phys Chem Chem Phys. 2013;15(36):14851-14855.

5. Ueno T, Nagano T. Fluorescent probes for sensing and imaging. Nat Methods. 2011;8(8):642-645.

6. Domaille DW, Que EL, Chang CJ. Synthetic fluorescent sensors for studying the cell biology of metals. Nat Chem Biol. 2008;4(3):168-175.

7. Urano Y, Sakabe M, Kosaka N. Rapid cancer detection by topically spraying a g-glutamyltranspeptidase-activated fluorescent probe. Sci Trans Med. 2011;3(110):110ra119.

8. Lavis LD, Raines RT. Bright ideas for chemical biology. ACS Chem Biol. 2008;3(3):142-155.

9. Wysockia LM, Lavis LD. Advances in the chemistry of small molecule fluorescent probes. Curr Opin Chem Biol. 2011;15(6):752-759.

10. Ashton TD, Jolliffeb KA, Pfeffer FM. Luminescent probes for the bioimaging of small anionic species in vitro and in vivo. Chem Soc Rev. 2015;44(14):4547-4595.

11. Loudet A, Burgess K. BODIPY dyes and their derivatives: syntheses and spectroscopic properties. Chem Rev. 2007;107(11):4891-4932.

12. Awuah SG, You Y. Boron dipyrromethene (BODIPY)-based photosensitizers for photodynamic Therapy. RSC $A d v$. 2012;2(30): 11169-11183.

13. Kamkaew A, Lim SH, Lee HL, Kiew LV, Chung LY, Burgess K. BODIPY dyes in photodynamic therapy. Chem Soc Rev. 2013;42(1):77-88.

14. Kowada T, Maedab H, Kikuchi K. BODIPY-based probes for the fluorescence imaging of biomolecules in living cells. Chem Soc Rev. 2015;44(14);4953-4972.

15. Nia Y, Wu J. Far-red and near infrared BODIPY dyes: synthesis and applications for fluorescent $\mathrm{pH}$ probes and bio-imaging. Org Biomol Chem. 2014;12(23):3774-3791.

16. Singh SP, Gayathri T. Evolution of BODIPY dyes as potential sensitizers for dye-sensitized solar cells. Eur J Org Chem. 2014;2014(22): 4689-4707.

17. Lu H, Mack J, Yanga Y, Shen Z. Structural modification strategies for the rational design of red/NIR region BODIPYs. Chem Soc Rev. 2014;43(13):4778-4823.

18. Ulrich G, Ziessel R, Harriman A. The chemistry of fluorescent Bodipy Dyes: versatility unsurpassed. Angew Chem Int Ed Engl. 2008;47(7) 1184-1201.

19. Benniston AC, Copley G. Lighting the way ahead with boron dipyrromethene (Bodipy) dyes. Phys Chem Chem Phys. 2009;11(21): 4124-4131.

20. Ziessel R, Ulricha G, Harriman A. The chemistry of Bodipy: a new El dorado for fluorescence tools. New J Chem. 2007;31(4):496-501.

21. Yuan L, Lin W, Zheng K, He L, Huang W. Far-red to near infrared analyteresponsive fluorescent probes based on organic fluorophore platforms for fluorescence imaging. Chem Soc Rev. 2013;42(2):622-661.

22. Boens N, Leen V, Dehaen W. Fluorescent indicators based on BODIPY. Chem Soc Rev. 2012;41(3):1130-1172.

23. Ziessel R, Harriman A. Artificial light-harvesting antennae: electronic energy transfer by way of molecular funnels. Chem Commun (Camb). 2011;47(2):611-631.

24. Wagner RW, Lindsey JS. A molecular photonic wire. J Am Chem Soc. 1994;116(21):9759-9760. 
25. Liu B, Li L, et al. Polyacetylenes containing BODIPY pendants with different connectivities: synthesis, characterization and opto-electronic properties. Polym Chem. 2014;5(2):372-381.

26. Wagner RW, Lindsey JS, Seth J, Palaniappan V, Bocian DF. Molecular Optoelectronic Gates. J Am Chem Soc. 1996;118(16):3996-3997.

27. Wagner RW, Lindsey JS. Boron-dipyrromethene dyes for incorporation in synthetic multi-pigment light-harvesting arrays. Pure Appl Chem. 1996;68(7):1373-1380.

28. Khan TK, Broring M, Mathur S, Ravikanth M. Boron dipyrrin-porphyrin conjugates. Coord Chem Rev. 2013;257(15-16):2348-2387.

29. Lakshmi V, Rao MR, Ravikanth M. Halogenated boron-dipyrromethenes: synthesis, properties and applications. Org Biomol Chem. 2015;13(9): 2501-2517.

30. Goud TV, Tutar A, Biellmann J. Synthesis of 8-heteroatom-substituted 4,4-difluoro-4-bora-3a,4a-diaza-s-indacene dyes (BODIPY). Tetrahedron. 2006;62(21):5084-5091.

31. Prokopcova H, Kappe CO. The Liebeskind-Srogl C-C cross-coupling reaction. Angew Chem Int Ed Engl. 2009;48(13):2276-2286.

32. Peña-Cabrera E, Aguilar-Aguilar A, Villanueva-Garcia F, et al. Simple, general, and efficient synthesis of meso-substituted borondipyrromethenes from a single platform. Org Lett. 2007;9(20):3985-3988.

33. Martinez-Gonzalez MR, Urías-Benavides A, Peña-Cabrera E, et al. Convenient access to carbohydrate-BODIPY hybrids by two complementary methods involving one-pot assembly of "clickable" BODIPY dyes. Eur J Org Chem. 2014;(26):5659-5663.

34. Arroyo IJ, Hu R, Peña-Cabrera E. et al. 8-Alkenylborondipyrromethene dyes. General synthesis, optical properties, and preliminary study of their reactivity. Tetrahedron. 2011;67(38):7244-7250.

35. Roachoa RI, Metta-Magañaa AJ, Peña-Cabrera E, Pannella KH. Synthesis, structural characterization, and spectroscopic properties of the ortho, meta, and para isomers of $8-\left(\mathrm{HOCH}_{2}-\mathrm{C}_{6} \mathrm{H}_{4}\right)$-BODIPY and 8-( $\left.\mathrm{MeOC}_{6} \mathrm{H}_{4}\right)$-BODIPY. J Phys Org Chem. 2013;26(4):345-351.

36. Arroyo IJ, Hu R, Merino G, Tang BZ, Peña-Cabrera E. The smallest and one of the brightest. Efficient preparation and optical description of the parent borondipyrromethene system. J Org Chem. 2009;74(15): 5719-5722.

37. Betancourt-Mendiola L, Valois-Escamilla I, Arbeloa T, et al. Scope and limitations of the Liebeskind-Srogl cross-coupling reactions involving the Biellmann BODIPY. J Org Chem. 2015;80(11):5771-5782.

38. Zhao N, Vicente GM, Fronczek FR, Smith KM. Synthesis of 3,8dichloro-6-ethyl-1,2,5,7-tetramethyl-BODIPY from an asymmetric dipyrroketone and reactivity studies at the 3,5,8-positions. Chemistry. 2015;21(16):6181-6192.

39. Gutierrez-Ramos BD, BaÇuelos J, Arbeloa T, et al. Straightforward synthetic protocol for the introduction of stabilized $\mathrm{C}$ nucleophiles in the BODIPY core for advanced sensing and photonic applications. Chemistry. 2015;21(4):1755-1764.

40. Osorio-Martínez CA, Urías-Benavides A, Gómez-Durán CF, et al. 8-AminoBODIPYs: cyanines or hemicyanines? the effect of the coplanarity of the amino group on their optical properties. J Org Chem. 2012;77(12):5434-5438.

41. Ma DH, Kim D, Akisawa T, Lee K, Kim K, Ahn KH. An FITC-BODIPY FRET couple: application to selective, ratiometric detection and bioimaging of cysteine. Chem Asian J. 2015;10(4):894-902.

42. Gomez-Duran CF, Garcia-Moreno I, Costela A, et al. 8-PropargylaminoBODIPY: unprecedented blue-emitting pyrromethene dye. Synthesis, photophysics and laser properties. Chem Commun. 2010;46(28): 5103-5105.

43. BaÇuelos J, Martin V, Gomez-Duran CF, et al. New 8-Amino-BODIPY derivatives: surpassing laser dyes at blue-edge wavelengths. Chemistry. 2011;17(26):7261-7270.

44. Esnal I, Urias-Benavides A, Gomez-Duran CF, et al. Reaction of amines with 8-methylthioBODIPY: dramatic optical and laser response to amine substitution. Chem Asian J. 2013;8(11):2691-2700.

45. Flores-Rizo JO, Esnal I, Osorio-Martínez CA, et al. 8-Alkoxy- and 8-aryloxy-BODIPYs: straightforward fluorescent tagging of alcohols and phenols. J Org Chem. 2013;78(12):5867-5877.
46. Esnal I, Valois-Escamilla I, Gómez-Durán CF, et al. Blue-to-orange color-tunable laser emission from tailored Boron-dipyrromethene dyes. Chemphyschem. 2013;14(18):4134-4142.

47. Leen V, Yuan P, Wang L, Boens N, Dehaen W. Synthesis of mesohalogenated BODIPYs and access to meso-substituted analogues. Org Lett. 2012;14(24):6150-6153.

48. Misra R, Dhokale B, Jadhav T, Mobin SM. Heteroatom-connected ferrocenyl BODIPYs: synthesis, structure, and properties. Organometallics. 2014;33(7):1867-1877. (b) Misra R, Dhokale B, Jadhav T, Mobin SM. Meso-Aryloxy and meso-arylaza linked BODIPY dimers: synthesis, structures and properties. New J Chem. 2014;38(8):3579-3585.

49. Boens N, Wang L, Leen V, et al. 8-HaloBODIPYs and their 8-(C, $\mathrm{N}, \mathrm{O}, \mathrm{S})$ substituted analogues: solvent dependent UV-Vis spectroscopy, variable temperature NMR, crystal structure determination, and quantum chemical calculations. $J$ Phys Chem A. 2014;118(9): $1576-1594$

50. Misra R, Dhokale B, Jadhav T, Mobin SM. The quenching of fluorescence as an indicator of donor-strength in meso arylethynyl BODIPY. Dalton Trans. 2014;43(12):4854-4861.

51. Dhokale B, Jadhav T, Mobin SM, Misra R. Meso enyne substituted BODIPYs: synthesis, structure and properties. Dalton Trans. 2015; 44(36): 15803-15812.

52. Misra R, Jadhav T, Dhokale B, et al. Carbazole-BODIPY conjugates: design, synthesis, structure and properties. Dalton Trans. 2014;43(34): 13076-13086.

53. Gibbs JH, Wang H, Bhupathiraju D, et al. Synthesis and properties of a series of carboranyl-BODIPYs. J Organomet Chem. Epub 2015.

54. Misra R, Dhokale B, Jadhav T, Mobin SM. Donor-acceptor mesoalkynylated ferrocenyl BODIPYs: synthesis, structure, and properties. Dalton Trans. 2013;42(37):13658-13666.

55. Wang H, Vicente GH, Fronczek FR, Smith KM. Synthesis and transformations of 5-Chloro-2,2'-dipyrrins and their boron complexes, 8-chloro-BODIPYs. Chemistry. 2014;20(17):5064-5074.

56. Misra R, Dhokale B, Jadhav T, Mobin SM. Donor-acceptor mesoalkynylated ferrocenyl BODIPYs: synthesis, structure, and properties. Dalton Trans. 2013;42(37):13658-13666.

57. Dhokale B, Jadhav T, Mobin SM, Misra R. Meso enamine substituted BODIPYs. Chem Commun (Camb). 2014;50(65):9119-9121.

58. Leen V, Leemans T, Boens N, Dehaen W. 2- and 3-Monohalogenated BODIPY dyes and their functionalized analogues: synthesis and spectroscopy. Eur J Org Chem. 2011;2011(23):4386-4396.

59. Duran-Sampedro G, Agarrabeitia AR, Garcia-Moreno I, et al. Chlorinated BODIPYs: surprisingly efficient and highly photostable laser dyes. Eur J Org Chem. 2012;2012(32):6335-6350.

60. Wu W, Sun J, Cui X, Zhao J. Observation of the room temperature phosphorescence of Bodipy in visible light-harvesting $\mathrm{Ru}$ (II) polyimine complexes and application as triplet photosensitizers for triplet-tripletannihilation upconversion and photocatalytic oxidation. JMater Chem C. 2013;1(30):4577-4589.

61. Jiao L, Pang W, Zhou J, et al. Regioselective stepwise bromination of boron dipyrromethene (BODIPY) Dyes. J Org Chem. 2011;76(24): 9988-9996.

62. Bozdemir ÖA, Büyükcakir O, Akkaya EU. Novel molecular building blocks based on the boradiazaindacene chromophore: applications in fluorescent metallosupramolecular coordination polymers. Chemistry. 2009;15(15):3830-3838.

63. Jiao L, Yu C, Li J, Wang Z, Wu M, Hao E. $\beta$-Formyl-BODIPYs from the Vilsmeier-Haack reaction. J Org Chem. 2009;74(19):7525-7528.

64. Esnal I, Bañuelos J, López Arbeloa I, et al. Nitro and amino BODIPYS: crucial substituents to modulate their photonic behavior. RSCAdv. 2013; 3(5):1547-1556.

65. Li L, Han J, Nguyen B, Burgess K. Syntheses and spectral properties of functionalized, water-soluble BODIPY derivatives. J Org Chem. 2008;73(5):1963-1970.

66. Hayashi Y, Yamaguchi S, Cha WY, Kim D, Shinokubo H. Synthesis of directly connected BODIPY oligomers through Suzuki-Miyaura coupling. Org Lett. 2011;13(12):2992-2995. 
67. Li Z, Chen Y, Lv X, Fu WF. A tetraphenylethene-decorated BODIPY monomer/dimer with intense fluorescence in various matrices. New $J$ Chem. 2013;37(11):3755-3761.

68. Majumdar P, Yuan X, Li S, et al. Cyclometalated Ir(III) complexes with styryl-BODIPY ligands showing near IR absorption/emission: preparation, study of photophysical properties and application as photodynamic/luminescence imaging materials. J Mater Chem B. 2014;2(19):2838-2854.

69. Cheng G, Fan J, Sun W, et al. A highly specific BODIPY-based probe localized in mitochondria for HClO imaging. Analyst. 2013;138(20): 6091-6096.

70. Saha S, Agarwalla H, Gupta H, et al. New chemodosimetric probe for the specific detection of $\mathrm{Hg}^{2+}$ in physiological condition and its utilisation for cell imaging studies. Dalton Trans. 2013;42(42):15097-15105.

71. Kolemen S, Cakmak Y, Kostereli Z, Akkaya EU. Atropisomeric dyes: axial chirality in orthogonal BODIPY oligomers. Org Lett. 2014;16(3): 660-663.

72. Khan TK, Ravikanth M. Synthesis of covalently linked borondipyrromethene-chromophore conjugates using 3-bromo borondipyrromethene as a key precursor. Tetrahedron. 2011;67(32): 5816-5824.

73. Haefele A, Zedde C, Retailleau P, Ulrich G, Ziessel R. Boron asymmetry in a BODIPY derivative. Org Lett. 2009;12(8):1672-1675.

74. Liras M, Bañuelos Prieto J, Pintado-Sierra M, et al. Synthesis, photophysical properties, and laser behavior of 3-amino and 3-acetamido BODIPY dyes. Org Lett. 2007;9(21):4183-4186.

75. Ganapathi E, Madhu S, Chatterjee T, Gonnade R, Ravikanth M. Synthesis, structure, spectral, electrochemical and sensing properties of 3-amino boron-dipyrromethene and its derivatives. Dyes Pigments. 2014;102:218-227.

76. Khan TK, Ravikanth M. 3-(Pyridine-4-thione)BODIPY as a chemodosimeter for detection of $\mathrm{Hg}(\mathrm{II})$ ions. Dyes Pigments. 2012;95(1):89-95.

77. Kand D, Mishra PK, Saha T, Lahiri M, Talukdar P. BODIPY based colorimetric fluorescent probe for selective thiophenol detection: theoretical and experimental studies. Analyst. 2012;137(17):3921-3924.

78. Wang L, Wang JW, Cui AJ, et al. Regioselective 2,6-dihalogenation of BODIPYs in 1,1,1,3,3,3-hexafluoro-2-propanol and preparation of novel meso-alkyl polymeric BODIPY dyes. RSC Adv. 2013;3(24):9219-9222.

79. Zhu S, Zhang J, Vegesna G, et al. Controlled Knoevenagel reactions of methyl groups of 1,3,5,7-tetramethyl BODIPY dyes for unique BODIPY dyes. RSC Adv. 2012;2(2):404-407.

80. Zhu S, Bi J, Vegesna G, et al. Functionalization of BODIPY dyes at 2,6positions through formyl groups. RSC Adv. 2013;3(14):4793-4800.

81. Shah M, Thangaraj K, Soong ML, et al. Pyrromethene-BF ${ }_{2}$ complexes as laser dyes: 1. Heteroatom Chem. 1990;1(5):389-399.

82. Krumova K, Cosa G. Bodipy dyes with tunable redox potentials and functional groups for further tethering: preparation, electrochemical, and spectroscopic characterization. J Am Chem Soc. 2010;132(49): 17560-17569.

83. Zhao H, Wang B, Liao J, Wang H, Tan G. Synthesis, spectral, electrochemical properties, and photovoltaic performance of structurally constrained BODIPY dyes with 4,4-dimethyltriphenylamine at the 2,6-positions. Tetrahedron Lett. 2013;54(45):6019-6022.

84. Baruah M, Qin W, Basarić N, De Borggraeve WM, Boens N. Bodipybased hydroxyaryl derivatives as fluorescent ph probes. J Org Chem. 2005;70(10):4152-4157.

85. Rao MR, Mobin SM, Ravikanth M. Boron-dipyrromethene based specific chemodosimeter for fluoride ion. Tetrahedron. 2010;66(9):1728-1734.

86. Rohand T, Baruah M, Qin W, Boens N, Dehaen W. Functionalisation of fluorescent BODIPY dyes by nucleophilic substitution. Chem Commun. 2006;(3):266-268.

87. Madhu S, Gonnade R, Ravikanth M. Synthesis of 3,5-bis(acrylaldehyde) boron-dipyrromethene and application in detection of cysteine and homocysteine in living cells. J Org Chem. 2013;78(10):5056-5060.

88. Yu C, Jiao L, Yin H, et al. $\alpha$-/ $\beta$-Formylated boron-dipyrrin (BODIPY) dyes: regioselective syntheses and photophysical properties. Eur J Org Chem. 2011;28:5460-5468.
89. Cieslik-Boczula K, Burgess K, Li L, et al. Photophysics and stability of cyano-substituted boradiazaindacene dyes. Photochem Photobiol Sci. 2009;8(7):1006-1015.

90. Khan TK, Ravikanth M. Synthesis of non-covalent BODIPYmetalloporphyrin dyads and triads. Tetrahedron. 2012;68(3):830-840.

91. Madhu S, Basu SK, Jadhav S, Ravikanth M. 3,5-Diformyl-borondipyrromethene for selective detection of cyanide anion. Analyst. 2013; 138(1):299-306.

92. Madhu S, Ravikanth M. Synthesis, spectral, electrochemical, and anion binding properties of 3,5-bis(dipyrromethanyl) boron-dipyrromethenes. Inorg Chem. 2012;51(7):4285-4292.

93. Madhu S, Bandela A, Ravikanth M. BODIPY based fluorescent chemodosimeter for explosive picric acid in aqueous media and rapid detection in the solid state. RSC Adv. 2014;4(14):7120-7123.

94. Madhu S, Ravikanth M. Boron-dipyrromethene based reversible and reusable selective chemosensor for fluoride detection. Inorg Chem. 2014;53(3):1646-1653.

95. Bill NL, Lim JM, Davis CM, et al. $\pi$-Extended tetrathiafulvalene BODIPY (ex-TTF-BODIPY): a redox switched "on-off-on" electrochromic system with two near-infrared fluorescent outputs. Chem Commun (Camb). 2014;50(51):6758-6761.

96. Leen V, Miscoria D, Yin S, et al. 1,7-Disubstituted boron dipyrromethene (BODIPY) dyes: synthesis and spectroscopic properties. J Org Chem. 2011;76(20):8168-8176.

97. Ortiz MJ, Agarrabeitia AR, Duran-Sampedro G, et al. Synthesis and functionalization of new polyhalogenated BODIPY dyes. Study of their photophysical properties and singlet oxygen generation. Tetrahedron. 2013;68(4):1153-1162.

98. Lakshmi V, Ravikanth M. Brominated boron dipyrrins: synthesis, structure, spectral and electrochemical properties. Dalton Trans. 2012;41(19):5903-5911.

99. Lakshmi V, Ravikanth M. Synthesis, spectral and electrochemical properties of phenylated boron-dipyrromethenes. Dyes and Pigm. 2013;96(3):665-671

100. Wakamiya A, Sugita N, Yamaguchi S. Red-emissive polyphenylated bodipy derivatives: effect of peripheral phenyl groups on the photophysical and electrochemical properties. Chem Lett. 2008;37(10): 1094-1095.

101. Lakshmi V, Ravikanth M. Synthesis of sterically crowded polyarylated boron-dipyrromethenes. J Org Chem. 2011;76(20):8466-8471.

102. Lakshmi V, Ravikanth M. Synthesis of hexasubstituted borondipyrromethenes having a different combination of substituents. Eur J Org Chem. 2014;26:5757-5766.

103. Lakshmi V, Ravikanth M. Polyarylated boron-dipyrromethenes containing three different types of aryl groups. RSC Adv. 2014;4(84): 44327-44336.

104. Ulrich G, Goze C, Guardigli M, Roda A, Ziessel R. Pyrromethene dialkynyl borane complexes for "cascatelle" energy transfer and protein labeling. Angew Chem Int Ed. 2005;44(24):3694-3698.

105. Goze C, Ulrich G, Ziessel R. Tetrahedral boron chemistry for the preparation of highly efficient "cascatelle" devices. J Org Chem. 2007;72(2):313-322.

106. Harriman A, Izzet G, Ziessel R. Rapid energy transfer in cascade-type bodipy dyes. J Am Chem Soc. 2006;128(33):10868-10875.

107. Goze C, Ulrich G, Ziessel R. Unusual fluorescent monomeric and dimeric dialkynyl dipyrromethene-borane complexes. Org Lett. 2006;8(20): 4445-4448.

108. Harriman A, Mallon LJ, Elliot KJ, et al. Length dependence for intramolecular energy transfer in three and four color donor-spacer-acceptor arrays. J Am Chem Soc. 2009;131(37):13375-13386.

109. Ulrich G, Goze C, Goeb S, Retailleaub P, Ziessel R. New fluorescent aryl- or ethynylaryl-boron-substituted indacenes as promising dyes. New J Chem. 2006;30(7):982-986.

110. Goze C, Ulrich G, Mallon LJ, Ziessel R. et al. Synthesis and photophysical properties of borondipyrromethene dyes bearing aryl substituents at the boron center. J Am Chem Soc. 2006;128(31): 10231-10239. 
111. Lu J, Ko S, Walters NR, Wang S. Decorating BODIPY with three- and four-coordinate boron groups. Org Lett. 2012;14(22):5660-5663.

112. Tahtaoui C, Thomas $\mathrm{C}$, Rohmer F, Hibert M. et al. Convenient method to access new 4,4-dialkoxyand 4,4-diaryloxy-diaza-s-indacene dyes: Synthesis and spectroscopic evaluation. J Org Chem. 2007;72(1): 269-272.

113. Brizet B, Eggenspiller A, Gros CP, et al. B,B-diporphyrinbenzyloxyBODIPY dyes: synthesis and antenna effect. J Org Chem. 2012;77(7): 3646-3650.

114. Hudnall TW, Gabba1 FP. A BODIPY boronium cation for the sensing of fluoride ions. Chem Commun (Camb). 2008;(38):4596-4597.
115. Bonnier C, Piers WE, Parvez M, Sorensen TS. Borenium cations derived from BODIPY dyes. Chem Commun (Camb). 2008;(38):4593-4595.

116. Lundrigan T, Crawford SM, Cameron TS, Thompson A. Cl-BODIPYs: a BODIPY class enabling facile B-substitution. Chem Commun (Camb). 2012;48(7):1003-1005.

117. Lundrigan T, Thompson A. Conversion of F-BODIPYs to ClBODIPYs: enhancing the reactivity of F-BODIPYs. J Org Chem. 2013; 78(2):757-761.

118. More AB, Mula S, Thakare $\mathrm{S}$, et al. Masking and demasking strategies for the $\mathrm{BF}_{2}$-BODIPYs as a tool for BODIPY fluorophores. J Org Chem. 2014;79(22):10981-10987.

\section{Publish your work in this journal}

Reports in Organic Chemistry is an international, peer-reviewed, open access journal publishing original research, reports, reviews and commentaries on all areas of organic chemistry. The manuscript management system is completely online and includes a very quick and fair peer-review system, which is all easy to use.

\section{Dovepress}

Visit http://www.dovepress.com/testimonials.php to read real quotes from published authors. 